\title{
Adaptive Multicarrier Modulation: A Convenient Framework for Time-Frequency Processing in Wireless Communications
}

\author{
THOMAS KELLER AND LAJOS HANZO
}

A historical perspective of orthogonal frequency-division multiplexing (OFDM) is given with reference to its literature. Its advantages and disadvantages are reviewed, and its performance is characterized over highly dispersive channels. The effects of both time- and frequency-domain synchronization errors are quantified, and a range of solutions proposed in the recent literature are reviewed. One of the main objectives of this review is to highlight the recent thinking behind adaptive bit allocation and turbo coding in the context of OFDM. This paper concludes with a wide-ranging throughput comparison of the schemes discussed herein under the unified constraint of a fixed target bit error rate of $10^{-4}$.

Keywords-Adaptive modulation, adaptive orthogonal frequency-division multiplexing (AOFDM), applications of OFDM, blind modem mode detection in OFDM, broadcasting using OFDM, channel quality estimation in OFDM, coded OFDM (COFDM), coherent and noncoherent detection of OFDM, crest factor in OFDM, frequency and timing errors in OFDM, HIPERLAN, mode signaling in OFDM, multicarrier modulation, OFDM, peak-to-mean envelope fluctuation in OFDM, phase noise in OFDM, preequalization, synchronization in OFDM, throughput of adaptive OFDM, wireless asynchronous transfer mode (WATM) using OFDM, wireless local-area networks (WLAN's) using OFDM.

\section{INTRODUCTION}

High-data-rate communications are limited not only by noise but-especially with increasing symbol rates-often more significantly by the intersymbol interference (ISI) due to the memory of the dispersive wireless communications channel [1]. Explicitly, this channel memory is caused by the dispersive channel impulse response (CIR) due to the different-length propagation paths between the transmitting and the receiving antennas. This dispersion effect could theoretically be measured by transmitting an infinitely short impulse and "receiving" the CIR itself. On this basis, several measures of the effective duration of the impulse response can be

Manuscript received September 2, 1999; revised February 2, 2000. This work was supported by EPSRC, U.K.; the Commission of the European Communities, Brussels, Belgium; and Motorola ECID, Swindon, U.K.

The authors are with the Department of Electronics and Computer Science, University of Southampton, Southampton SO17 1BJ U.K. (e-mail: lh@ecs.soton.ac.uk).

Publisher Item Identifier S 0018-9219(00)04564-3. calculated, one being the delay spread. The multipath propagation of the channel manifests itself by different echos of possibly different transmitted symbols overlapping at the receiver, which leads to error rate degradation.

This effect occurs not only in wireless communications but also over all types of electrical and optical waveguides, although for these media the relative time differences are comparatively small, mostly due to multimode transmission or incorrect electrical or optical termination at interfaces.

In wireless communications systems, the duration and the shape of the CIR depend heavily on the propagation environment of the communications system in question. While indoor wireless networks typically exhibit only short relative delays, outdoor networks, like the global system of mobile communications [2], can face delay spreads on the order of $15 \mu \mathrm{s}$.

As a general rule, the effects of ISI on the transmission-error statistics are negligible as long as the delay spread is significantly shorter than the duration of one transmitted symbol. This implies that the symbol rate of communications systems is practically limited by the channel's memory. For higher symbol rates, there is typically significant deterioration of the system's error rate performance.

If symbol rates exceeding this limit are to be transmitted over the channel, mechanisms must be implemented in order to combat the effects of ISI. Channel equalization techniques [1] can be used to suppress the echoes caused by the channel. In order to perform this operation, the CIR must be estimated. Significant research efforts were invested into the development of such channel equalizers, and most wireless systems in operation use equalizers to combat ISI.

There is, however, an alternative approach toward transmitting data over a multipath channel. Instead of attempting to cancel the effects of the channel's echos, orthogonal frequency-division multiplexing (OFDM) [1] modems employ a set of subcarriers in order to transmit information symbols in parallel-in so-called subchannels-over the channel. Since the system's data throughput is the sum of all the parallel channels' throughputs, the data rate per subchannel is only a fraction of the data rate of a conven- 
tional single-carrier system having the same throughput. This allows us to design a system supporting high data rates while maintaining symbol durations much longer than the channel's memory, thus circumventing the need for channel equalization.

The outline of this paper is as follows. Section II commences with a historical perspective on OFDM, highlighting the associated research issues with reference to the literature. Based on the above overview of the state-of-the-art, Section III characterizes the performance of OFDM over dispersive, wide-band channels, while Section IV quantifies the effects of synchronization errors on OFDM, leading to Section $\mathrm{V}$, which highlights the range of synchronization solutions proposed by the research community at large. Again commencing with a literature survey, the key topic of adaptive bit allocation over highly frequency-selective wireless channels is the subject of Section VI, while Section VII is dedicated to the closely related subject of preequalization and channel coding. Our discourse is concluded in Section VIII with a wide-ranging throughput comparison of the schemes discussed in the paper under the unified constraint of a fixed target bit error rate (BER) of $10^{-4}$.

\section{ORThogOnAl FREQUENCY-DiVISION MULTIPLEXING}

\section{A. Historical Perspective}

Frequency-division multiplexing or multitone systems have been employed in military applications since the 1960's - for example, by Bello [3], Zimmerman [4], Powers and Zimmerman [5], and others. Orthogonal frequency-division multiplexing, which employs multiple carriers overlapping in the frequency domain, was pioneered by Chang [6], [7]. Saltzberg [8] studied a multicarrier system employing orthogonal time-staggered quadrature amplitude modulation (O-QAM) on the carriers.

The use of the discrete Fourier transform (DFT) to replace the banks of sinusoidal generators and the demodulators-suggested by Weinstein and Ebert [9] in 1971—significantly reduces the implementation complexity of OFDM modems. This substantial implementational complexity reduction was attributable to the simple realization that the DFT uses a set of harmonically related sinusoidal and cosinusoidal basis functions, whose frequency is an integer multiple of the lowest nonzero frequency of the set, which is referred to as the basis frequency. These harmonically related frequencies can hence be used as the set of carriers required by the OFDM system. For a formal proof of this, the interested reader is referred to [1].

In 1980, Hirosaki [10] suggested an equalization algorithm in order to suppress both intersymbol and intersubcarrier interference (ISCI) caused by the CIR or timing and frequency errors. Simplified OFDM modem implementations were studied by Peled [1] in 1980, while Hirosaki [12] introduced the DFT-based implementation of Saltzberg's O-QAM OFDM system. Kolb [13], Schüßler [14], Preuss [15], and Rückriem [16] conducted further research into the application of OFDM. Kalet [17] introduced the concept of allocating more bits to subcarriers, which were, for example, near the center of the transmission frequency band and hence were less attenuated than those near the edge of the transmission band. However, since Kalet's discussions were cast in the context of slowly varying channels, the concept of near-instantaneously adaptive transmission was not introduced at this early stage of OFDM research. This concept was often referred to as "water-filling" in the frequency domain. A few years later, Cimini [18] provided early seminal results on the performance of OFDM modems in mobile communications channels.

More recent advances in OFDM transmission are presented in the impressive state-of-art collection of works edited by Fazel and Fettweis [19], including research by Fettweis et al., Rohling et al., Vandendorp et al., Lindner et al., Kammeyer et al., and Meyr et al. [20], [21], but the impressive individual contributions are too numerous to mention.

While OFDM transmissions over mobile communications channels can alleviate the problem of multipath propagation, recent research efforts have focused on solving a set of inherent difficulties regarding OFDM, namely, on reducing the associated the peak-to-mean-power ratio fluctuation, on time and frequency synchronization and on mitigating the effects of cochannel interference sensitivity in multiuser environments. These issues are addressed below in more depth.

1) Peak-to-Mean Power Ratio: It is plausible that the OFDM signal—which is the superposition of a high number of modulated subchannel signals-may exhibit a high instantaneous signal peak with respect to the average signal level. Furthermore, large signal amplitude swings are encountered, when the time-domain signal traverses from a low instantaneous power waveform to a high-power waveform. Similarly, the peak-to-mean power envelope fluctuates dramatically, when traversing the origin upon switching from one phasor to another. Both of these events may result in a high out-of-band (OOB) harmonic distortion power, unless the transmitter's power amplifier exhibits an extremely high linearity [1] across the entire signal dynamic range. This potentially contaminates the adjacent channels with adjacent channel interference. Practical amplifiers exhibit a finite amplitude range, in which they can be considered near linear. In order to prevent severe clipping of the high OFDM signal peaks - which is the main source of OOB emissions - the power amplifier must not be driven into saturation and hence they are typically operated with a certain so-called backoff, creating a "head-room" for the signal peaks, which reduces the risk of amplifier saturation and OOB emission. Two different families of solutions have been suggested in the literature, in order to mitigate these problems, either reducing the peak-to-mean power ratio or improving the amplification stage of the transmitter.

More explicitly, Shepherd [22], Jones [23], and Wulich [24] suggested different coding techniques that aim to minimize the peak power of the OFDM signal. According to their approach, different data encoding or mapping schemes are employed before modulation. A simple example is concatenating a number of dummy bits to a string of information bits with the sole aim of mitigating the so-called Crest 
factor or peak-to-mean signal envelope ratio. Müller [25], Pauli [26], May [27], and Wulich [28] suggested different algorithms for postprocessing the time-domain OFDM signal prior to amplification, while Schmidt and Kammeyer [29] employed adaptive subcarrier allocation in order to reduce the Crest factor. Dinis and Gusmão [30]-[32] researched the use of two-branch amplifiers, while the so-called clustered OFDM technique introduced by Daneshrad et al. [33] operates with a set of parallel partial fast Fourier transform (FFT) processors with associated transmitting chains. More explicitly, clustered OFDM allows a number of users to share a given bandwidth amongst a number of users on a demand basis, potentially supporting a peak data rate identical to that of a single-user OFDM system. The bandwidth assigned to a particular user is typically constituted by a number of subcarrier clusters, which are spread sufficiently far apart from each other, in order to provide frequency diversity. OFDM systems with increased robustness to nonlinear distortion have been proposed-for example, by Okada et al.[34] as well as by Dinis and Gusmão [35].

2) Synchronization: Time and frequency synchronization between the transmitter and receiver are of crucial importance in terms of the performance of an OFDM link [36]-[40]. A wide variety of techniques has been proposed for estimating and correcting both timing and carrier-frequency offsets at the OFDM receiver. Rough timing and frequency acquisition algorithms relying on known pilot symbols or pilot tones embedded into the OFDM symbols have been suggested by Claßen [20], Warner [41], Sari [42], and Moose [43], as well as Brüninghaus and Rohling [44]. Fine frequency and timing tracking algorithms exploiting the OFDM signal's cyclic extension were published by Moose [43], Daffara [45], and Sandell [46].

3) $O F D M / C D M A$ : Combining OFDM transmissions with code division multiple access (CDMA) allows us to exploit the wide-band channel's inherent frequency diversity by spreading each symbol across multiple subcarriers. This technique has been pioneered by Yee et al. [47], by Chouly et al. [48], and by Fettweis et al. [49]. Fazel and Papke [50] investigated convolutional coding in conjunction with OFDM/CDMA. Prasad and Hara [51] compared various methods of combining the two techniques, identifying three different structures, namely multicarrier CDMA (MC-CDMA), multicarrier direct-sequence CDMA (MC-DS-CDMA), and multitone CDMA (MT-CDMA). Like nonspread OFDM transmission, OFDM/CDMA methods suffer from high peak-to-mean power ratios, which are dependent on the frequency-domain spreading scheme, as has been investigated by Choi et al. [52].

4) Adaptive Antennas: Combining adaptive antenna techniques with OFDM transmissions was shown to be advantageous in suppressing cochannel interference in cellular communications systems. Li et al. [53]-[56], Kim et al. [57], and Münster et al. [58] have investigated algorithms for multiuser channel estimation and interference suppression. The employment of adaptive antennas is always beneficial in terms of mitigating the effects of multiuser interference, since with the aid of beam-steering it becomes possible to focus the receiver's antenna beam on the served user, while attenuating the cochannel interferers. This is of particularly high importance in conjunction with OFDM, which exhibits a high sensitivity against cochannel interference, potentially hampering its application in cochannel interference limited multiuser scenarios.

5) OFDM Applications: Due to their implementational complexity, OFDM applications have been scarce until quite recently. Recently, however, OFDM has been adopted as the new European digital audio broadcasting (DAB) standard [59]-[63], as well as for the terrestrial digital video broadcasting (DVB-T) system [42], [64]. The hostile propagation environment of the terrestrial system requires concatenated Reed-Solomon [2] (RS) and rate-compatible punctured convolutional coding [2] (RCPCC) combined with OFDM. These schemes are capable of delivering high-definition video at bit rates of up to $20 \mathrm{Mbits} / \mathrm{s}$ in slowly time-varying broadcast-mode distributive wireless scenarios. Recently, a range of DVB system performance studies were also published in the literature [65]-[68], portraying the DVB-T system.

For fixed-wire applications, OFDM is employed in the asynchronous digital subscriber line (ADSL) and high-bit-rate DSL (HDSL) systems [69]-[72], and it has also been suggested for power-line communications systems [73], [74] due to its resilience to time-dispersive channels and narrow-band interferers.

More recently, OFDM applications were studied within the European Fourth Framework Advanced Communications Technologies and Services (ACTS) program [75]. Specifically, the Pan-European Median project investigated a 155Mbit/s (Mb/s) wireless asynchronous transfer mode (WATM) network [76]-[79], while the Magic WAND group [80], [81] developed a wireless local-area network (LAN). Hallmann and Rohling [82] presented a range of different OFDM-based systems that were applicable to the European Telecommunication Standardization Institute's (ETSI) third-generation air interface [83].

Lastl, the recently standardized high performance local-area network standard known as HIPERLAN/2 was designed for providing convenient wireless networking in indoor environments and also invoked OFDM. The wireless provision of high-bit-rate services appears a more attractive alternative than installing wireline based networks. The HIPERLAN standard specifies the air interface and the physical layer, in order to ensure the compatibility of different manufacturers' equipment, while refraining from standardizing the higher layer functions of the system. The HIPERLAN standard constitutes a member of the broad-band radio access networks family often referred to as BRAN [84], [85]. The BRAN family of recommendations is constituted by the HIPERLAN/1 and / 2 systems operating in the $5-\mathrm{GHz}$ frequency band. Further members of the family include the so-called HIPERACCESS standard contrived for fixed wireless broad-band point-to-multipoint access and the HIPERLINK recommendation designed for wireless broad-band communications in the $17-\mathrm{GHz}$ frequency band. The system's parameters are summarized in Table 1. 
Table 1

HIPERLAN/2 Physical Layer Parameters [86]

\begin{tabular}{l|l}
\hline No. of subcarriers (SC) & 64 \\
\hline No. of active SCs & 52, where 48 are used for \\
data and 4 for pilots \\
\hline Channel spacing & $20 \mathrm{MHz}$ \\
\hline Sampling rate & $20 \mathrm{Msample/s}$ \\
\hline Guard interval & $800 \mathrm{~ns}$ or 16 time-domain \\
\hline SC modulation & samples \\
\hline Demodulation & BPSK, QPSK, 16QAM \\
\hline Channel coding & and $64 \mathrm{QAM}$ \\
\hline & coherent \\
\hline rate $1 / 2$ convolutional \\
\hline
\end{tabular}

\section{B. OFDM Modem Structure}

The principle of any FDM system is to split the information to be transmitted into $N$ parallel streams, each of which modulates a carrier using an arbitrary modulation technique. The frequency spacing between adjacent carriers is $\Delta f$, resulting in a total signal bandwidth of $N \cdot \Delta f$. The resulting $N$ modulated and multiplexed signals are transmitted over the channel, and at the receiver $N$ parallel receiver branches recover the information. A multiplexer then recombines the $N$ parallel information streams into a high-rate serial stream.

The conceptually simplest implementation of an FDM modem is to employ $N$ independent transmitter/receiver pairs, which is often prohibitive in terms of complexity and cost [12]. Weinstein [9] suggested the digital implementation of FDM subcarrier modulators/demodulators based on the DFT.

The DFT and its more efficient implementation, the FFT, are employed for the base-band OFDM modulation/demodulation process, as can be seen in the schematic shown in Fig. 1. The associated harmonically related frequencies can hence be used as the set of subchannel carriers required by the OFDM system. However, instead of carrying out the modulation/demodulation on a subcarrier by subcarrier basis, as in Hirosaki's early proposal, for example [10], all OFDM subchannels are modulated/demodulated in a single inverse DFT

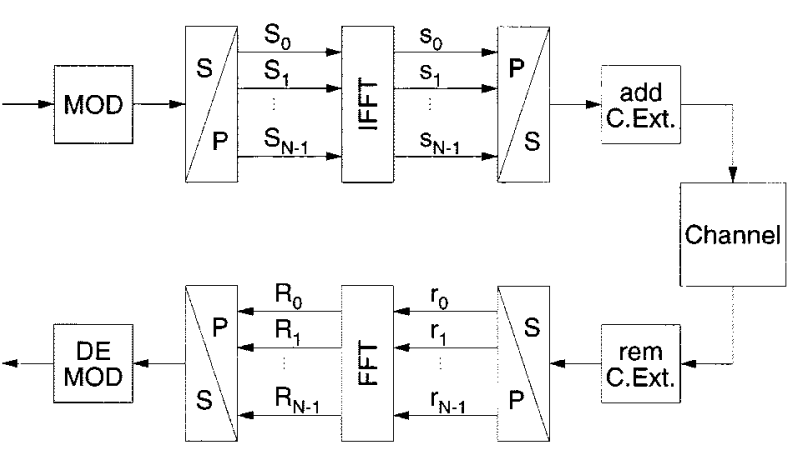

Fig. 1. Schematic of $N$-subcarrier OFDM transmission system.

(IDFT)/DFT step. For more detailed explanations and signal waveforms, the interested reader is referred to [1].

The serial data stream is mapped to data symbols with a symbol rate of $1 / T_{s}$, employing a general phase and amplitude modulation scheme, and the resulting symbol stream is demultiplexed into a vector of $N$ data symbols $S_{0}$ to $S_{N-1}$. The parallel data symbol rate is $1 / N \cdot T_{s}$, i.e., the parallel symbol duration is $N$ times longer than the serial symbol duration $T_{s}$. Hence the effects of the dispersive channel—which are imposed on the transmitted signal as the convolution of the signal with the CIR-become less damaging, affecting only a fraction of the extended signaling pulse duration. The inverse FFT of the data symbol vector is computed and the coefficients $s_{0}$ to $s_{N-1}$ constitute an OFDM symbol, as seen in the figure. Since the harmonically related and modulated individual OFDM subcarriers can be conveniently visualized as the spectrum of the signal to be transmitted, it is the IFFT - rather than the FFT - which is invoked, in order to transform the signal's spectrum to the time domain for transmission over the channel. The associated modulated signal samples $s_{n}$ are the time-domain samples of the OFDM symbol and are transmitted sequentially over the channel at a symbol rate of $1 / T_{s}$. At the receiver, a spectral decomposition of the received time-domain samples $r_{n}$ is computed employing an $N$-tap FFT, and the recovered data symbols $R_{n}$ are restored in serial order and demultiplexed, as seen in Fig. 1.

The underlying assumption in the context of OFDM upon invoking the IFFT for modulation is that although $N$ frequency-domain samples produce $N$ time-domain samples, both signals are assumed to be periodically repeated over an infinite time-domain and frequency-domain interval, respectively. In practice, however, it is sufficient to repeat the time-domain signal periodically for the duration of the channel's memory, i.e., for a duration that is comparable to the length of the CIR. This is, namely, the time interval required for the channel's transient response to die down after exciting the channel with a time-domain OFDM symbol. Once the channel's transient response time has elapsed, its output is constituted by its steady-state response constituted by the received time-domain OFDM symbol. In order to ensure that the received time-domain OFDM symbol is demodulated from the channel's steady-state-rather than from its transient-response, each time-domain OFDM symbol is extended by the so-called cyclic extension (C. Ext. 


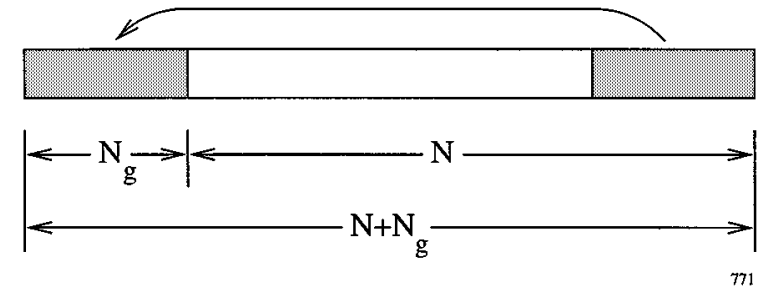

Fig. 2. Stylized plot of $N$-subcarrier OFDM time-domain signal with a cyclic extension of $N_{g}$ samples.

in Fig. 1) or guard interval of $N_{g}$ samples duration, in order to overcome the inter-OFDM symbol interference due to the channel's memory. The signal samples received during the guard interval are discarded at the receiver and the $N$-sample received time-domain OFDM symbol is deemed to follow the guard interval of $N_{g}$ samples duration. The demodulated OFDM symbol is then generated from the remaining $N$ samples upon invoking the IFFT. We note, however, that since the transmitted time-domain signal was windowed to the finite duration of $N+N_{g}$ samples, the corresponding transmitted frequency-domain signal is convolved with the sinc-shaped frequency-domain transfer function of the rectangular time-domain window function. As a result of this frequency-domain convolution, the originally pure line-spectrum of the IFFT's output generates a sinc-shaped subchannel spectrum centered on each OFDM subcarrier.

The samples of the cyclic extension are copied from the end of the time-domain OFDM symbol, generating the transmitted time-domain signal $\left(s_{N-N_{g}-1}, \ldots, s_{N-1}\right.$, $\left.s_{0}, \ldots, s_{N-1}\right)$ depicted in Fig. 2. At the receiver, the samples of the cyclic extension are discarded. Clearly, the need for a cyclic extension in time-dispersive environments reduces the efficiency of OFDM transmissions by a factor of $N /\left(N+N_{g}\right)$. Since the duration $N_{g}$ of the necessary cyclic extension depends only on the channel's memory, OFDM transmissions employing a high number of carriers $N$ are desirable for efficient operation. Typically, a guard interval length of not more than $10 \%$ of the OFDM symbol's duration is employed. Again, for further details concerning the operation of OFDM modems, please refer to [87], [1], [88].

\section{Modulation in the Frequency Domain}

Modulation of the OFDM subcarriers is analogous to the modulation in conventional serial systems. The modulation schemes of the subcarriers are generally quadrature amplitude modulation or phase shift keying (PSK) [1] in conjunction with both coherent and noncoherent detection. Differentially coded star-QAM (DSQAM) [1] can also be employed. If coherently detected modulation schemes are employed, then the reference phase of the OFDM symbol must be known, which can be acquired with the aid of pilot tones [89] embedded in the spectrum of the OFDM symbol, as will be discussed in Section III. For differential detection the knowledge of the absolute subcarrier phase is not necessary, and differentially coded signaling can be invoked either between neighboring subcarriers or between the same subcarriers of consecutive OFDM symbols.

\section{OFDM TRANSMISSION OVER FREQUENCY-SELECTIVE CHANNELS}

\section{A. System Parameters}

Based on the above advances in the field of OFDM modems, below we will characterize the expected performance of OFDM modems using the example of high-rate wireless asynchronous transfer mode (WATM) systems [76]-[78], [80], [81]. Specifically, the system parameters used in characterizing the performance of various OFDM algorithms closely followed the specifications of the ACTS Median system [76]-[79], which is a proposed wireless extension to fixed-wire ATM-type networks. In the Median system, the OFDM FFT length is 512, and each symbol is padded with a cyclic prefix of length 64 . The sampling rate

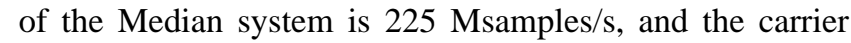
frequency is $60 \mathrm{GHz}$. The uncoded target data rate of the Median system is $155 \mathrm{Mb} / \mathrm{s}$.

OFDM modems were originally conceived in order to transmit data reliably in time-dispersive or frequency-selective channels without the need for a complex time-domain channel equalizer. In this contribution, the techniques employed for the transmission of QAM OFDM signals over a time-dispersive channel are discussed and channel estimation methods are investigated [1].

\section{B. The Channel Model}

The channel model assumed in this paper is that of a finite impulse response (FIR) filter with time-varying tap values. Every propagation path $i$ is characterized by a fixed delay $\tau_{i}$ and a time-varying amplitude $A_{i}(t)=a_{i} \cdot g_{i}(t)$, which is the product of a complex amplitude $a_{i}$ and a Rayleigh fading process $g_{i}(t)$. The Rayleigh processes $g_{i}$ are independent from each other, but they all exhibit the same normalized Doppler frequency $f_{d}^{\prime}$.

The ensemble of the $p$ propagation paths constitutes the impulse response

$$
h(t, \tau)=\sum_{i=1}^{p} A_{i}(t) \cdot \delta\left(\tau-\tau_{i}\right)=\sum_{i=1}^{p} a_{i} \cdot g_{i}(t) \cdot \delta\left(\tau-\tau_{i}\right)
$$

which is convolved with the transmitted signal.

The channel model employed in this contribution is the worst case operating environment for an indoor wireless ATM network similar to that of the ACTS Median system [76]-[79]. We assumed a vehicular velocity of about $50 \mathrm{~km} / \mathrm{h}$ or $13.9 \mathrm{~m} / \mathrm{s}$, resulting in a normalized Doppler frequency of $f_{d}^{\prime}=1.235 \cdot 10^{-5}$. We note here that the normalized Doppler frequency in this contribution was related to the OFDM symbol duration rather than to the time-domain signal's sample duration. This relationship will be formally defined in (5). Hence, suffice to say here that the normalized Doppler frequency in this sense is typically 512 times lower than the conventional normalized Doppler frequency due to 


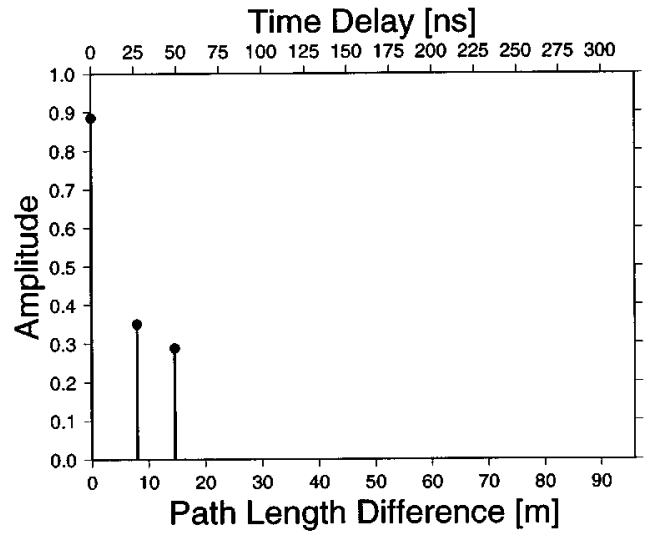

(a)
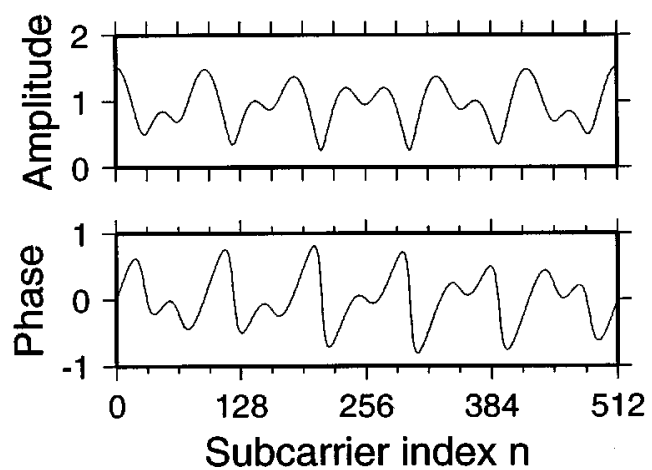

(b)

Fig. 3. WATM channel: (a) impulse response (b) frequency-domain channel transfer function $H(n)$ experienced by a specific OFDM symbol.

having 512 samples per PFDM symbol. The significance of this will become more clear in the context of adaptive OFDM schemes, where the predictability of the channel's frequency-domain transfer function between consecutive OFDM symbols depends explicitly on the duration of the symbol.

The vehicular velocity of $50 \mathrm{~km} / \mathrm{h}$ constitutes the highest possible speed of for example an indoor forktruck in a warehouse environment. Again, this worst case speed was employed in order to provide performance results characterizing the worst possible scenario in the context of adaptive OFDM transceivers, which are sensitive to rapid CIR or transfer function variations. This issue will become more explicit during our further discourse. The impulse response was determined by simple ray-tracing in a warehouse-type environment and is shown in Fig. 3(a), where each CIR tap corresponds to a specifically delayed propagation path. We note that this indoor CIR is not particularly dispersive; however, at the $155-\mathrm{Mb} / \mathrm{s}$ WATM rate, the dispersion corresponds to 11 sample periods, which would require a high-performance channel equalizer in a serial modem.

The last CIR path arrives at a delay of $48.9 \mathrm{~ns}$ due to the reflection with an excess path length of about $15 \mathrm{~m}$ with respect to the line-of-sight path, which again, corresponds to 11 sample periods. The impulse response exhibits a root mean squared (RMS) delay spread of $1.5276 \cdot 10^{-8} \mathrm{~s}$ and is shown in Fig. 3(a). The resulting frequency-domain transfer function for this WATM impulse response is given in Fig. 3(b), which exhibits an undulating behavior across the 512 subcarriers. This suggests that the high-quality subcarrier may be able to use several bits per subcarrier, while others may have to be disabled. This issue will be further detailed during our later discourse.

\section{Effects of Time-Dispersive Channels}

The effects of the time-variant and time-dispersive channels on the data symbols transmitted in an OFDM symbol's subcarriers are diverse. First, if the impulse response of the channel is longer than the duration of the OFDM guard interval, then energy will spill over between consecutive OFDM symbols, leading to inter-OFDM-symbol interfer- ence. We will not elaborate on these effects here, since the length of the guard interval is generally chosen to be longer than the longest anticipated CIR.

If the channel is changing only slowly compared to the duration of an OFDM symbol, then a near time-invariant CIR can be associated with each transmitted OFDM symbol, which, however, slightly changes between consecutive OFDM symbols. In this case, the frequency-selective transfer function of the channel results in a frequency-dependent multiplicative distortion of the received frequency-domain OFDM symbols. This frequency-domain phenomenon is somewhat analogous to the time-domain effects of a time-domain fading channel envelope in a serial or single-carrier modem.

Let us now briefly view the system in the time domain again. The role of the guard interval was discussed in depth before. Hence, suffice to state here that if the CIR duration is shorter than the OFDM guard interval, then no inter-OFDM-symbol interference is experienced. More explicitly, if the "memory" or the "echoes" of the dispersive CIR have died down during the guard interval, i.e., before the commencement of the information-bearing OFDM symbol section, the consecutive OFDM symbols will not interfere with each other. This scenario is analogous to a narrow-band or nondispersive fading channel in the context of a serial modem. This will be elaborated on in Section III-C1. A rapidly time-varying channel, however, will introduce intersubcarrier interference due to the channel's time-variant impulse response. The effects of this will be studied in Section III-C2.

1) Effects of the Slowly Time-Varying Time-Dispersive Channel: Here a channel is referred to as slowly time-varying if the CIR does not vary significantly over the duration of one OFDM symbol, but it is time-variant over longer periods of time. In this case, the time-domain convolution of the transmitted time-domain signal with the CIR corresponds simply to the multiplication of the spectrum of the signal with the channel's frequency-domain transfer function $H(f)$, as seen in (2)

$$
s(t) * h(t) \longleftrightarrow S(f) \cdot H(f)
$$




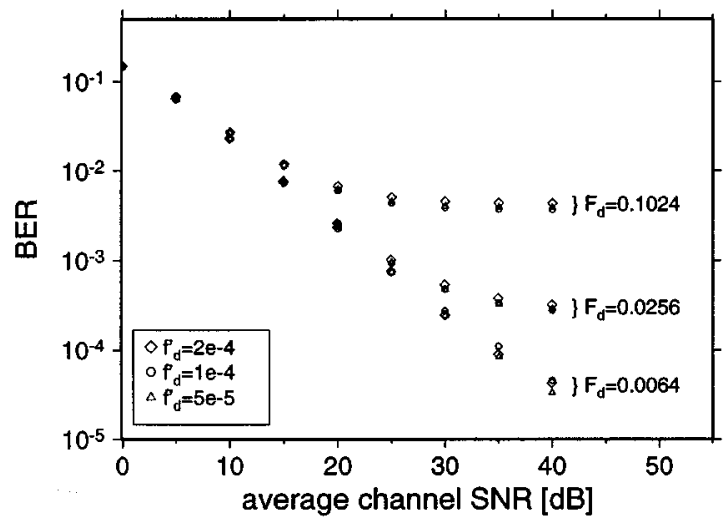

(a)

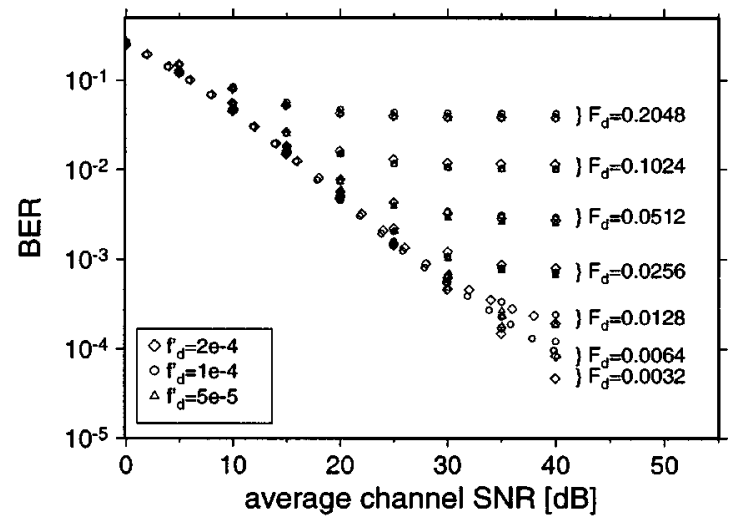

(b)

Fig. 4. BPSK OFDM modem performance in a fading narrow-band channel for normalized Doppler frequencies of $f_{d}^{\prime}=5 \cdot 10^{-5}, 1 \cdot 10^{-4}$, and $2 \cdot 10^{-4}$ and FFT lengths between 16 and 4096, where $F_{d}=f_{d} \cdot N T_{s}=f_{d}^{\prime} \cdot N$.

where the channel's frequency-domain transfer function $H(f)$ is the Fourier transform of the impulse response $h(t)$

$$
h(t) \longleftrightarrow H(f) .
$$

Since the information symbols $S(n)$ are encoded into the amplitude of the transmitted spectrum at the subcarrier frequencies $f_{n}$, the received symbols $R(n)$ are the product of the transmitted symbol with the channel's frequency-domain transfer function $H(n)$ plus the additive complex Gaussian noise samples $N(n)$

$$
R(n)=S(n) \cdot H(n)+N(n) .
$$

We note here that the additive time-domain noise imposed by the channel becomes correlated due to the filtering effect of the demodulator's FFT operation. Let us now consider the effects of rapidly time-varying channels.

2) Rapidly Time-Varying Channel: A channel is classified here as rapidly time-varying if the CIR changes significantly over the duration of an OFDM symbol. In this case, the frequency-domain transfer function is time-variant during the transmission of an OFDM symbol and this timevarying frequency-domain transfer function leads to the loss of orthogonality between the OFDM symbol's subcarriers. The amount of this intersubcarrier interference depends on the rate of change in the impulse response.

The simplest environment to study the effects of rapidly time-varying channels is the narrow-band channel, whose impulse response consists of only one fading path. If the amplitude of this path is varying in time, then the received OFDM symbol's spectrum will be the original OFDM spectrum convolved with the spectrum of the channel variation during the transmission of the OFDM symbol. Since this short-term channel spectrum is varying between different OFDM transmission bursts, the effects of the time-varying narrow-band channel have to be averaged over a high number of transmission bursts for the sake of arriving at reliable performance estimates.
Since the interference is caused by the variation of the CIR during the transmission of each OFDM symbol, we introduce the "OFDM-symbol normalized" Doppler frequency $F_{d}$

$$
F_{d}=f_{d} \cdot N T_{s}=f_{d}^{\prime} \cdot N
$$

where

$$
\begin{array}{ll}
N & \text { FFT length; } \\
1 / T_{s} & \text { sampling rate; } \\
f_{d} & \text { Doppler frequency characterizing the fading } \\
& \text { channel; }
\end{array}
$$

$f_{d}^{\prime}=f_{d} \cdot T_{s}$ conventional normalized Doppler frequency.

The BER performance for an OFDM modem for a set of different FFT lengths and different channel Doppler frequencies was determined by simulation, and the simulation results for binary phase shift keying (BPSK) are given in Fig. 4. Fig. 4(a) depicts the BER performance of an OFDM modem employing BPSK with perfect narrow-band fading channel estimation, where it can be observed that for any given value of $F_{d}$ the different FFT lengths and channels behave similarly. For an $F_{d}$ value of 0.0256 , a residual BER of about 2.8 $\cdot 10^{-4}$ is observed, while for $F_{d}=0.1024$ the residual BER is about $0.37 \%$, where-again- $F_{d}=f_{d} \cdot N T_{s}=f_{d}^{\prime} \cdot N$.

3) Signaling over Time-Dispersive OFDM Channels: Analogously to the case of serial modems in narrow-band fading channels, the amplitude and phase variations inflicted by the channel's frequency-domain transfer function $H(n)$ upon the received symbols will severely affect the bit-error probabilities, where different modulation schemes suffer to different extents from the effects of the channel transfer function. Coherent modulation schemes rely on the knowledge of the symbols' reference phase, which will be distorted by the phase of $H(n)$. Hence if such a modulation scheme is to be employed, then this phase distortion has to be estimated and corrected. For multilevel modulation schemes [1], where the magnitude of the received symbol also bears information, the magnitude of $H(n)$ will affect the demodulation. Clearly, the performance of such a system depends on the quality of the channel estimation.

A simpler approach to signaling over fading channels is to employ differential modulation, where the information is 
encoded in the difference between the individual modulated symbols mapped to consecutive subcarriers of the OFDM symbol. Differential phase shift keying (DPSK) employs the phase of the previous modulated symbol conveyed by the previous subcarrier as phase reference, encoding information in the phase difference between consecutive modulated symbols. DPSK is thus only affected by the differential channel phase distortion between two consecutive symbols assigned to consecutive subcarriers, rather than by the channel phase distortion's absolute value. We note here that differential encoding between the corresponding identical-frequency subcarriers of consecutive OFDM symbols could also be invoked, although the associated channel phase change would be more substantial and hence the former approach to differential encoding is more advantageous.

\section{OFDM PERFORMANCE WITH FREQUENCY AND TIMING ERRORS}

In this section, we will highlight the effects of timeand frequency-domain synchronization errors on the performance of an OFDM system. Furthermore, a number of synchronization algorithms will be briefly highlighted for time-domain burst-based OFDM communications systems based on the recent advances in the literature.

The performance of the synchronization subsystem, in particular, the accuracy of the frequency and timing error estimations, is of major influence on the overall OFDM system performance. In order to demonstrate the effects of carrier frequency and time-domain FFT window alignment errors, a series of results will be presented over different channels. For all the additive white Gaussian noise (AWGN) channel experiments rectangular time-domain pulse shaping was assumed.

\section{A. Effects of Frequency Shift on OFDM}

Carrier frequency errors result in a shift of the received signal's spectrum in the frequency domain. If the frequency error is an integer multiple $I$ of the subcarrier spacing $\Delta f$, then the received frequency domain subcarriers are shifted by $I \cdot \Delta f$. The subcarriers are still mutually orthogonal, but the received data symbols, which were mapped to the OFDM spectrum, are in the wrong position in the demodulated spectrum, resulting in a BER of 0.5 .

If the carrier frequency error is not an integer multiple of the subcarrier spacing, then energy is spilling over between the subcarriers, resulting in loss of their mutual orthogonality. In other words, interference is observed between the subcarriers, which deteriorates the BER of the system. The amount of this intersubcarrier interference can be evaluated by observing the spectrum of the OFDM symbol.

The spectrum of the OFDM signal is derived from its timedomain representation transmitted over the channel. A single OFDM symbol in the time domain can be described as

$$
u(t)=\left[\sum_{n=0}^{N-1} a_{n} e^{j \omega_{n} \cdot t}\right] \times \operatorname{rect}\left(\frac{t}{N \cdot T_{s}}\right)
$$

which is the sum of $N$ subcarriers $e^{\omega_{n} \cdot t}$, each modulated by a QAM symbol $a_{n}$ and windowed by a rectangular window of the OFDM symbol duration $T_{s}$. The Fourier transform of this rectangular window is a frequency-domain sinc-function, which is convolved with the dirac-delta subcarriers, determining the spectrum of each of the windowed complex exponential functions, leading to the spectrum of the $n$th single subcarrier in the form of

$$
A_{n}(\omega)=\frac{\sin \left(N \cdot T_{s} \cdot \omega / 2\right)}{N \cdot T_{s} \cdot \omega / 2} * \delta\left(\omega-\omega_{n}\right) .
$$

Replacing the radian frequencies $\omega$ by frequencies and using the relationship $N \cdot T_{s}=1 / \Delta f$, the spectrum of a subcarrier can be expressed as

$$
A_{n}(f)=\frac{\sin \left(\pi \frac{f-f_{n}}{\Delta f}\right)}{\pi \frac{f-f_{n}}{\Delta f}}=\operatorname{sinc}\left(\frac{f-f_{n}}{\Delta f}\right) .
$$

The OFDM receiver samples the received time-domain signal, demodulates it by invoking the FFT, and-in case of a carrier frequency shift-generates the subchannel signals in the frequency domain at the sampling points $f_{n}+\delta f$. These sampling points are spaced from each other by the subcarrier spacing $\Delta f$ and misaligned by the frequency error $\delta f$. This scenario is shown in Fig. 5. Fig. 5(a) shows the sampling of the subcarrier at frequency $f_{n}$ at the optimum frequency raster, resulting in a maximum signal amplitude and no intersubcarrier interference. If the frequency reference of the receiver is offset with respect to that of the transmitter by a frequency error of $\delta f$, then the received symbols suffer from intersubcarrier interference, as depicted in Fig. 5(b).

The total amount of intersubcarrier interference experienced by subcarrier $n$ is the sum of the interference amplitude contributions of all the other subcarriers in the OFDM symbol

$$
I_{n}=\sum_{j, j \neq n} a_{j} \cdot A_{j}\left(f_{n}+\delta f\right) .
$$

Since the QAM symbols $a_{j}$ are random variables, the interference amplitude in subcarrier $n, I_{n}$, is also a random variable, which cannot be calculated directly. If the number of interferers is high, however, then the power spectral density of $I_{n}$ can be approximated with that of a Gaussian process, according to the central limit theorem. Therefore, the effects of the intersubcarrier interference can be modeled by additional white Gaussian noise superimposed on the frequency-domain data symbols.

The variance of this Gaussian process $\sigma$ is the sum of the variances of the interference contributions

$$
\sigma^{2}=\sum_{j, j \neq n} \sigma_{a_{j}}^{2} \cdot\left|A_{j}\left(f_{n}+\delta f\right)\right|^{2} .
$$

The quantities $\sigma_{a_{j}}^{2}$ are the variances of the data symbols, which are the same for all $j$ in a system that is not varying the average symbol power across different subcarriers. Additionally, because of the constant subcarrier spacing of $\Delta f$, the 
$A(f)$

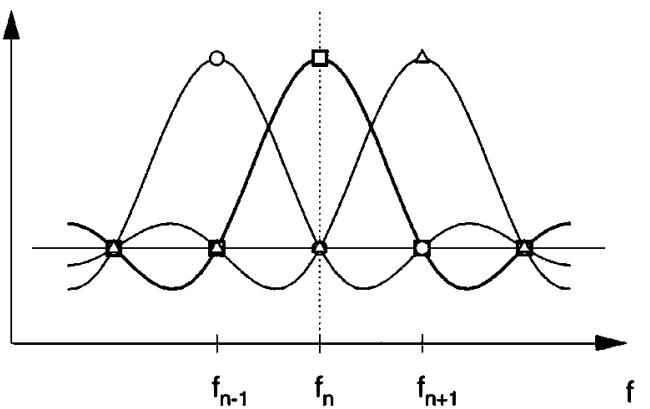

(a)
$A(f)$

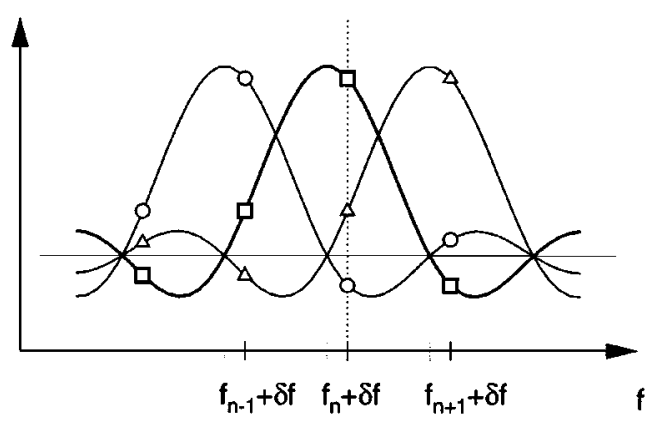

(b)

Fig. 5. Stylized plot of OFDM symbol spectrum with sampling points for three subcarriers. The symbols on the curves signify the contributions of the three subcarriers to the sum at the sampling point: (a) no frequency offset between transmitter and receiver (b) frequency error $\delta f$ present.

interference amplitude contributions can be expressed more conveniently as

$$
A_{j}\left(f_{n}+\delta f\right)=A_{j} n(\delta f)=\operatorname{sinc}\left((n-j)+\frac{\delta f}{\Delta f}\right) .
$$

The sum of the interferer powers leads to the intersubcarrier interference variance expression of

$$
\sigma^{2}=\sigma_{a}^{2} \cdot \sum_{i=-N / 2-1}^{N / 2}\left|\operatorname{sinc}\left(i+\frac{\delta f}{\Delta f}\right)\right|^{2} .
$$

The value of the ISCI variance for FFT lengths of $N=$ 64,512 , and 4096 and for a range of frequency errors $\delta f$ is shown in Fig. 6. It can be seen that the number of subcarriers does not influence the ISCI noise variance for OFDM symbol lengths of more than 64 subcarriers. This is due to the rapid decrease of the interference amplitude with increasing frequency separation, so that only the interference from close subcarriers contributes significantly to the interference load on the subcarriers.

In order to quantify the accuracy of the Gaussian approximation, histograms of the measured interference amplitude were produced for quadrature phase shift keying (QPSK) and 16-QAM modulation of the subcarriers. The triangles in Fig. 7 depict the histograms of ISCI noise magnitudes recorded for a 512-subcarrier OFDM modem employing QPSK and 16-QAM in a system having a frequency error of $\delta f=0.3 \Delta f$. The continuous line drawn on the same graph is the corresponding approximation of the histogram by a Gaussian probability density function (pdf) of the variance calculated using (12). It can be observed that the Gaussian curve is a reasonable approximation for both histograms in the central region, but that for the tails of the distributions the Gaussian function exhibits high relative errors. The histogram of the interference caused by the 16-QAM signal is, however, closer to the Gaussian curve than the QPSK interference histogram.

The frequency mismatch between the transmitter and receiver of an OFDM system not only results in intersubcarrier interference but also reduces the useful signal amplitude at the frequency-domain sampling point by a factor of $f(\delta f)=\operatorname{sinc}(\delta f / \Delta f)$. Using this and $\sigma^{2}$, the theoretical influence of the intersubcarrier interference, approximated by a Gaussian process, can be calculated for a given modulation

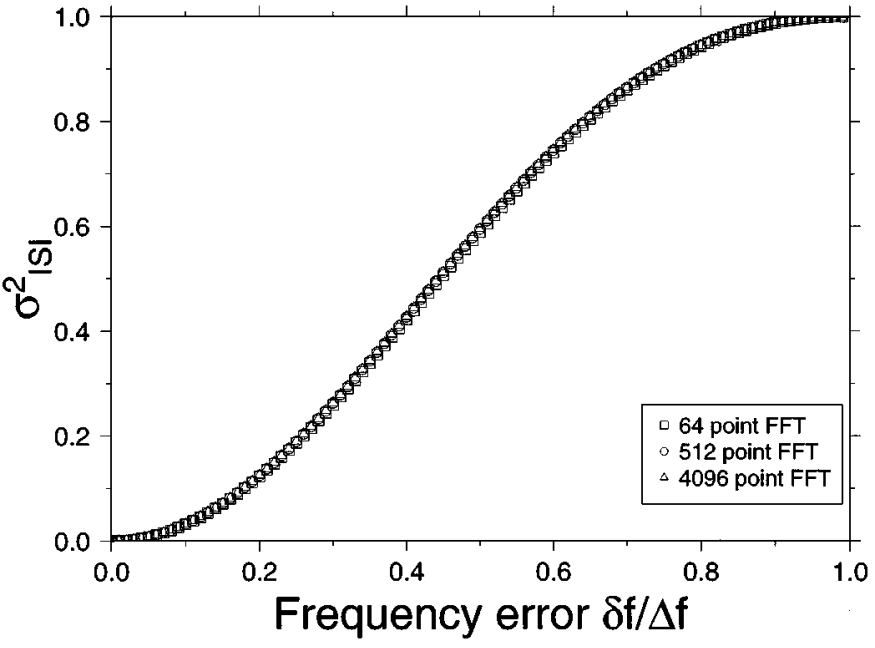

Fig. 6. Intersubcarrier interference variance due to a frequency shift $\delta f$ FFT lengths of $N$ of 64,512 , and 4096, for normalized frequency errors $\delta f / \Delta f$ between zero and one.

scheme in a AWGN channel. In the case of coherently detected QPSK, the closed-form expression for the BER $P_{e}(\gamma)$ at a channel signal-to-noise ratio (SNR) $\gamma$ is given by [90]

$$
P_{e}(\gamma)=Q(\sqrt{\gamma})
$$

where the Gaussian $Q()$-function is defined as [90]

$$
Q(y)=\frac{1}{\sqrt{2 \pi}} \int_{y}^{\infty} e^{-x^{2} / 2} d x=\frac{1}{2} \operatorname{erfc}\left(\frac{y}{\sqrt{2}}\right) .
$$

Assuming that the effects of the frequency error can be approximated by white Gaussian noise of variance $\sigma^{2}$ and taking into account the attenuated signal magnitude $f(\delta f)=$ $\operatorname{sinc}(\pi \delta f / \Delta f)$, we can adjust the equivalent SNR to

$$
\gamma^{\prime}=\frac{f(\delta f) \cdot \sigma_{a}^{2}}{\sigma^{2}+\sigma_{a}^{2} / \gamma}
$$

where $\sigma_{a}^{2}$ is the average symbol power and $\gamma$ is the real channel SNR. Comparison between the theoretical BER calculated using $\gamma^{\prime}$ and QPSK simulation results for frequency errors of $\delta f=0.15 \Delta f$ and $0.2 \Delta f$ are shown in Fig. 8(a). While for both frequency errors the theoretical BER using the Gaussian approximation fits the simulation results well for channel SNR values of up to $12 \mathrm{~dB}$, the predictions and the simulation results diverge for higher values of SNR. The 


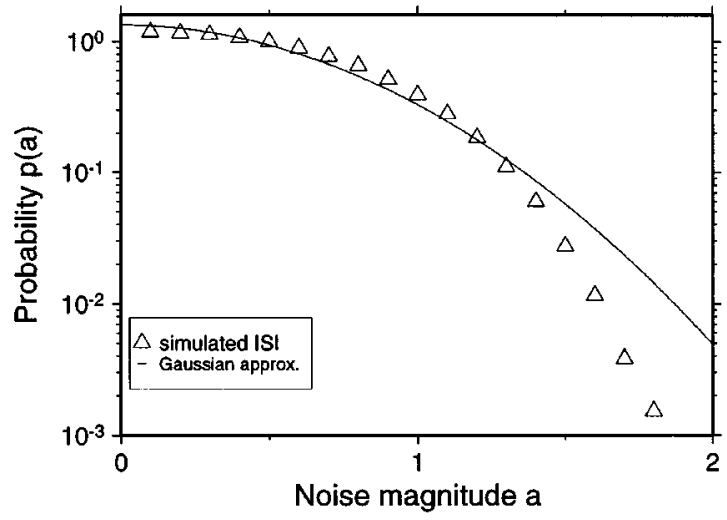

(a)

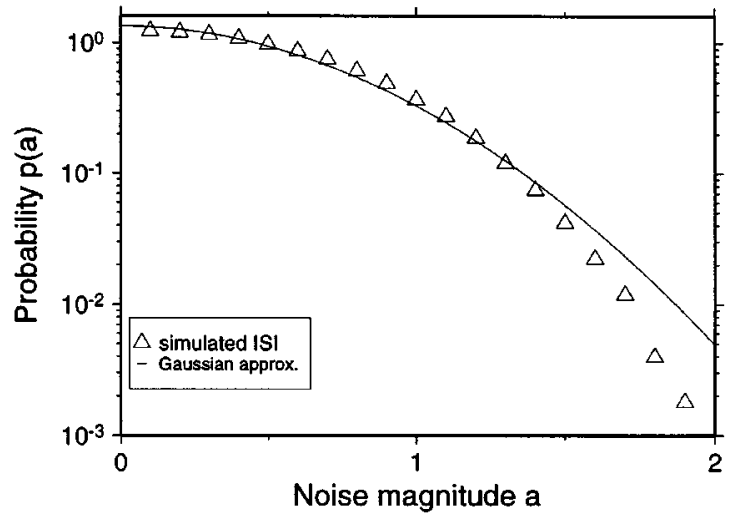

(b)

Fig. 7. Histogram of the ISCI magnitude for a simulated 512-subcarrier OFDM modem using QPSK or 16-QAM for $\delta f=0.3 \Delta f$; the line represents the Gaussian approximation having the same variance.

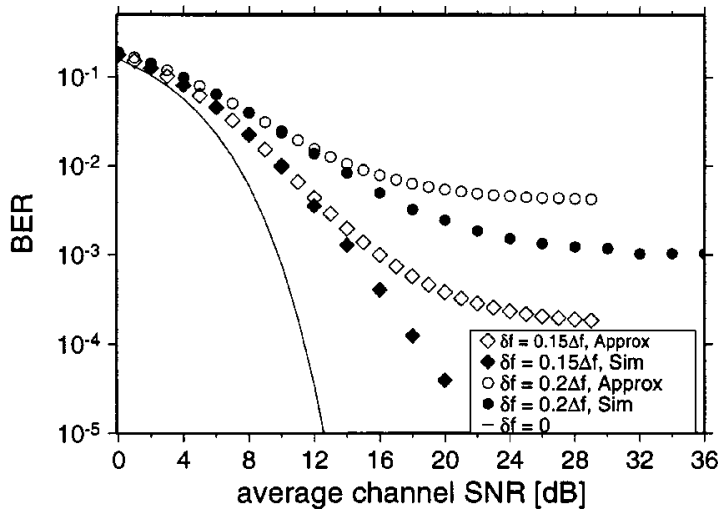

(a)

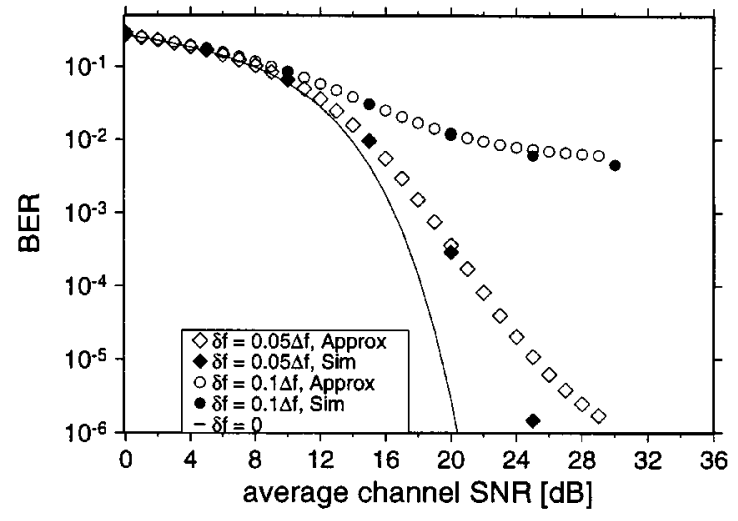

(b)

Fig. 8. The effect of intersubcarrier interference due to frequency synchronization error on the BER over AWGN channels. (a) Bit-error probability versus channel SNR for frequency errors of $0.15 \Delta f$ and $\Delta f$ for a QPSK modem. (b) BER versus channel SNR for frequency errors of $0.05 \Delta f$ and $0.1 \Delta f$ for a 16-QAM modem. In both graphs, the black markers are simulated BER results, while the white markers are the predicted BER curves using the Gaussian intersubcarrier interference model.

pessimistic BER prediction is due to the pronounced discrepancy between the histogram and the Gaussian curve in Fig. 7 at the tail ends of the amplitude histograms, since for high noise amplitudes the Gaussian model is a poor approximation for the intersubcarrier interference.

The equivalent experiment-conducted for coherently detected 16-QAM-results in the simulated and predicted BER's depicted in Fig. 8(b). For 16-QAM transmission, the noise resilience is much lower than for QPSK. Hence for these experiments, smaller values of $\delta f=0.05 \Delta f$ and $0.1 \Delta f$ have been chosen. It can be observed that the Gaussian noise approximation is a much better fit for the simulated BER in a 16-QAM system than for a QPSK modem. This is in accordance with Fig. 7, where the histograms of the interference magnitudes were depicted.

\section{B. Effect of Time-Domain Synchronization Errors on OFDM}

Unlike frequency mismatch, as discussed above, time synchronization errors do not result in intersubcarrier interference. Instead, if the receiver's FFT window spans samples from two consecutive OFDM symbols, inter-OFDM-symbol interference occurs.

Additionally, even small time-domain misalignments of the FFT window result in an evolving phase shift in the frequency-domain symbols, leading to BER degradation. Initially, we will concentrate on these phase errors.

If the receiver's FFT window is shifted with respect to that of the transmitter, then the time shift property of the Fourier transform, formulated as

$$
\begin{aligned}
f(t) & \longleftrightarrow F(\omega) \\
f(t-\tau) & \longleftrightarrow e^{-j \omega \tau} F(\omega)
\end{aligned}
$$

describes its effects on the received symbols. Any misalignment $\tau$ of the receiver's FFT window will introduce a phase error of $2 \pi \Delta f \tau / T_{s}$ between two adjacent subcarriers. If the time shift is an integer multiple $m$ of the sampling time $T_{s}$, then the phase shift introduced between two consecutive subcarriers is $\delta \phi=2 \pi m / N$, where $N$ is the FFT length employed. This evolving phase error has a considerable influence on the BER performance of the OFDM system, clearly depending on the modulation scheme used. 


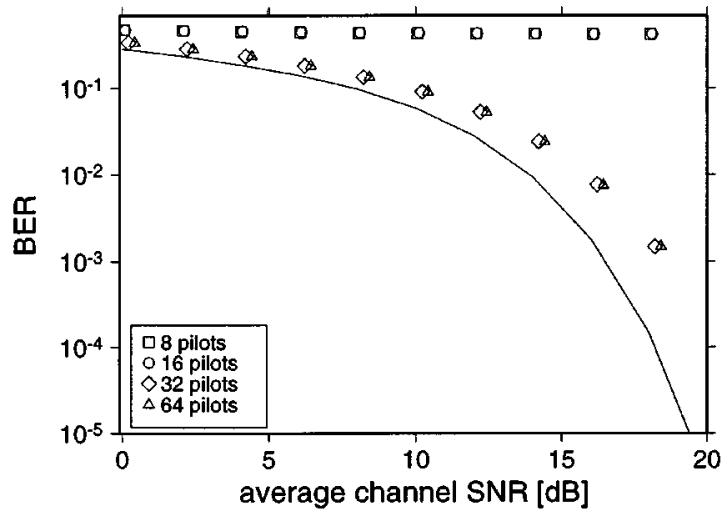

(a)

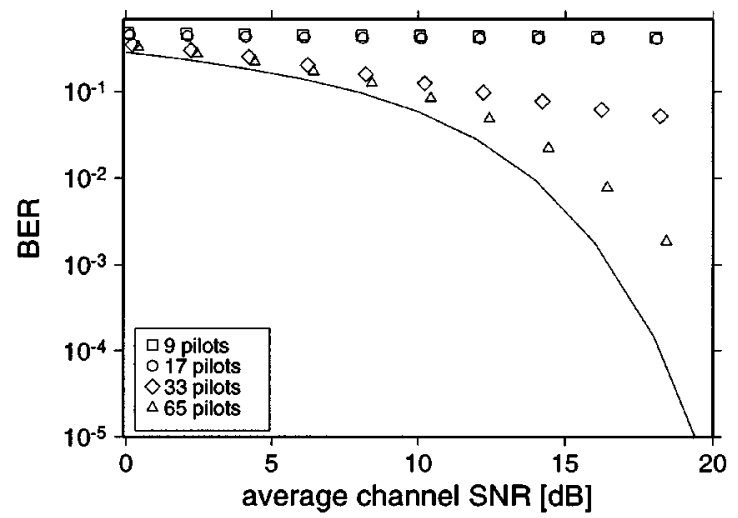

(b)

Fig. 9. BER versus channel SNR performance for 16-level PSA-QAM in an AWGN channel for different pilot subcarrier spacings in the presence of a fixed FFT window misalignment of $\tau=10 T_{s}$. The OFDM FFT length is 512. (a) PSAM interpolation using ideal low-pass interpolator. (b) PSAM using linear interpolator. In both graphs, the line marks the coherently detected 16-QAM performance in absence of both FFT window misalignment and PSAM.

1) Coherent Modulation: Coherent modulation schemes suffer the most from FFT window misalignments, since the reference phase evolves by $2 \pi$ throughout the frequency range for every sampling time misalignment $n \cdot T_{s}$. Clearly, this results in a total loss of the reference phase, and hence coherent modulation cannot be employed without phase correction mechanisms, if imperfect time synchronization is to be expected.

2) Pilot Symbol Assisted Modulation: Pilot-symbol-assisted-modulation (PSAM) schemes [89], [1] can be employed in order to mitigate the effects of spectral attenuation and the phase rotation throughout the FFT bandwidth. Pilots are interspersed with the data symbols in the frequency domain and the receiver can estimate the evolving phase error from the received pilots' phases.

The number of pilot subcarriers necessary for correctly estimating the channel transfer function depends on the maximum anticipated time shift $\tau$. Following the notion of the frequency-domain channel transfer function $H(n)$ introduced in Section III, the effects of phase errors can be written as

$$
H(f)=e^{-j 2 \pi f \tau} .
$$

Replacing the frequency variable $f$ by the subcarrier index $n$, where $f=n \Delta f=n /\left(N T_{s}\right)$ and normalizing the time misalignment $\tau$ to the sampling time $T_{s}$, so that $\tau=m$. $T_{s}$, the frequency-domain channel transfer function can be expressed as

$$
H(n)=e^{-j 2 \pi(n m / N)} .
$$

The number of pilots necessary for correctly estimating this frequency-domain channel transfer function $H(n)$ is dependent on the normalized time delay $m$. Following the Nyquist sampling theorem, the distance $\Delta p$ between two pilot tones in the OFDM spectrum must be less than or equal to half the period of $H(n)$, so that

$$
\Delta p \leq \frac{N}{2 m} \text {. }
$$

The simulated performance of a 512-subcarrier 16-QAM PSAM modem in the presence of a constant timing error of $\tau=10 T_{s}$ in an AWGN channel is depicted in Fig. 9 for both ideal low-pass and for simple linear interpolation. Following (20), the maximum acceptable pilot subcarrier distance required for resolving a normalized FFT-window misalignment of $m=\tau / T_{s}=10$ is $\Delta p=N / 20=512 / 20=25.6$, requiring at least 20 pilot subcarriers equidistantly spaced in the OFDM symbol. We can see in both graphs of Fig. 9 that the BER is 0.5 for both schemes, if fewer than 20 pilot subcarriers are employed in the OFDM symbol. For pilot numbers above the required minimum of 20 , however, the performance of the ideal low-pass interpolated PSAM scheme does not vary with the number of pilots employed, while the linearly interpolated PSAM scheme needs higher numbers of pilot subcarriers in order to achieve a similar performance. The continuous lines in the graphs show the BER for a coherently detected 16-QAM OFDM modem in the absence of timing errors, while utilizing no PSAM. Observe in the figure that there is a BER penalty for PSAM in a narrow-band AWGN channel, since the pilots are affected by noise, which is interpreted by the PSAM schemes as a channel-induced fluctuation, which has to be compensated.

3) Differential Modulation: As stated before, differential encoding [1] can be implemented both between corresponding subcarriers of consecutive OFDM symbols or between adjacent subcarriers of the same OFDM symbol. The latter was found more advantageous, since there is less channel-induced-rather than modulation-induced-phase rotation between consecutive subcarriers of an OFDM symbol than between the identical-frequency subcarriers of consecutive OFDM symbols. Hence differential encoding between adjacent subcarriers was employed here. Simulations have been performed for a 512-subcarrier OFDM system, employing DBPSK and DQPSK for different FFT window misalignment values.

The BER performance curves for timing errors of up to six positive and negative sampling intervals are displayed in Fig. 10. The figure suggests that in case of time-advanced 


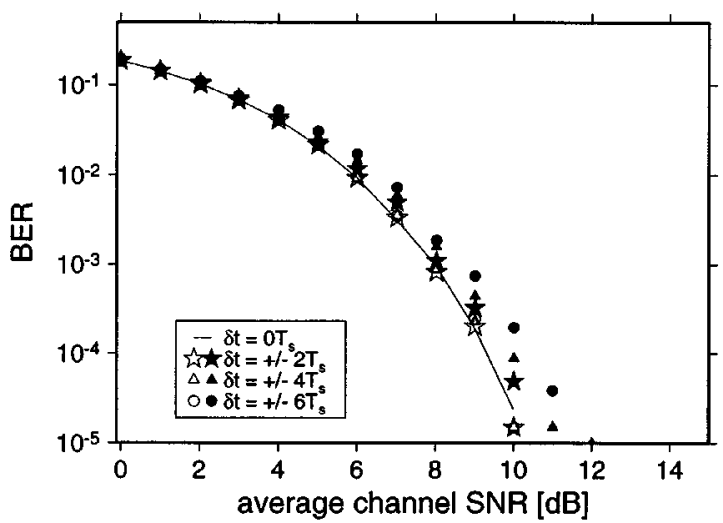

(a)

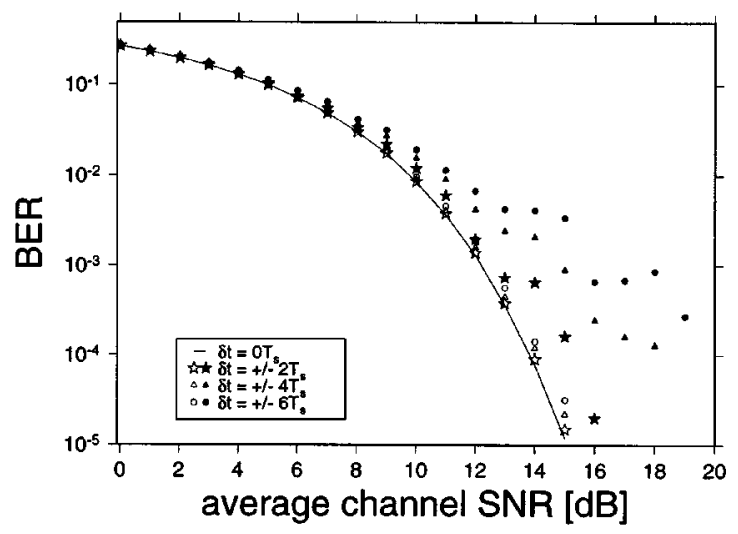

(b)

Fig. 10. BER versus SNR over AWGN channels for a 512-subcarrier OFDM modem employing DBPSK and DQPSK, respectively. Positive time shifts imply time-advanced FFT window or delayed received data.

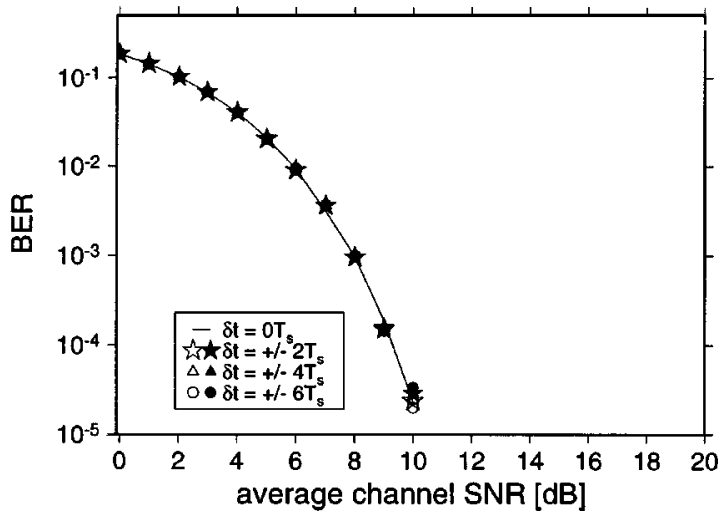

(a)

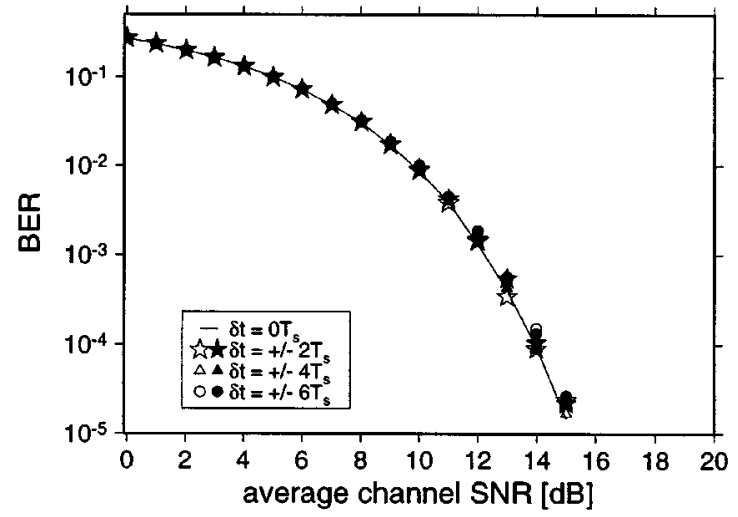

(b)

Fig. 11. BER versus SNR over AWGN channels for a 512-subcarrier OFDM modem employing a postamble of ten symbols for DBPSK and DQPSK, respectively. Positive time shifts correspond to time-advanced FFT window or delayed received data.

data (bold markers in the figure) or time-delayed FFT windows, the BER degrades due to including samples of the previous OFDM symbol in the current FFT window, while neglecting some of samples belonging to the current OFDM symbol. This data-dependent error is the reason for the fluctuating BER in the figure. Note that one sample interval misalignment represents a phase error of $2 \pi / 512$ between two consecutive samples, which explains why the BER effects of the simulated positive timing misalignments marked by the hollow symbols are negligible for DBPSK. Specifically, a maximum SNR degradation of $0.5 \mathrm{~dB}$ was observed for DQPSK.

Positive FFT window time shifts correspond to a delayed received data stream, and hence all samples in the receiver's FFT window belong to the same quasi-periodically extended OFDM symbol. In the case of negative time shifts, however, the effects on the BER are much more severe due to interOFDM-symbol interference. Since the data are received prematurely, the receiver's FFT window contains samples of the forthcoming OFDM symbol, not from the cyclic extension of the wanted symbol. This scenario can only be encountered in conjunction with imperfect OFDM symbol synchronization, when the OFDM symbols are received prematurely.
This nonsymmetrical behavior of the OFDM receiver with respect to positive and negative relative timing errors can be mitigated by adding a short postamble, consisting of copies of the OFDM symbol's first samples. Fig. 11 shows the BER versus SNR curves for the same offsets, while using a tensample postamble. Now, the behavior for positive and negative timing errors becomes symmetrical. Clearly, the required length of this postamble depends on the largest anticipated timing error, which adds further redundancy to the system. This postamble can be usefully employed, however, to make an OFDM system more robust to time misalignments and thus to simplify the task of the time-domain FFT window synchronization system.

\section{SyNCHRONIZATION ALGORITHMS}

The results of Section IV indicate that the accuracy of a modem's time- and frequency-domain synchronization system dramatically influences the overall BER performance. We have seen that carrier frequency differences between the transmitter and the receiver of an OFDM system will introduce additional impairments in the frequency domain caused by inter-subcarrier interference, while FFT 
window misalignments in the time domain will lead to phase errors between the subcarriers. Both of these effects will degrade the system's performance and have to be kept to a minimum by the synchronization system.

In a time-division multiple access (TDMA)-based OFDM system, the frame synchronization between a master station-in cellular systems generally the base station - and the portable stations has to be also maintained. For these systems, a so-called reference symbol marking the beginning of a new time frame is commonly used. This added redundancy can be exploited for both frequency synchronization and FFT-window alignment, if the reference symbol is correctly chosen.

In order to achieve synchronization with a minimal amount of computational effort at the receiver, while also minimizing the amount of redundant information added to the data signal, the synchronization process is normally split into a coarse acquisition phase and a fine tracking phase, if the characteristics of the random frequency and timing errors are known. In the acquisition phase, an initial estimate of the errors is acquired, using more complex algorithms and possibly a higher amount of synchronization information in the data signal, whereas later the tracking algorithms only have to correct for small short-term deviations.

At the commencement of the synchronization process, neither the frequency error nor the timing misalignment are known; hence synchronization algorithms must be found that are sufficiently robust to initial timing and frequency errors. In the forthcoming sections, we will briefly review the associated literature before providing some performance figures for the sake of illustration.

\section{A. Coarse Transmission Frame and OFDM Symbol Synchronization}

Coarse frame and symbol synchronization algorithms presented in the literature all rely on additional redundancy inserted in the transmitted data stream. The Pan-European DVB system uses a so-called null-symbol as the first OFDM symbol in the time frame, during whose duration no energy is transmitted [91], and which is detected by monitoring the received baseband power in the time domain, without invoking FFT processing. Claßen [20] proposed an OFDM synchronization burst of at least three OFDM symbols per time frame. Two of the OFDM symbols in the burst would contain synchronization subcarriers bearing known symbols along with normal data transmission carriers, but one of the OFDM symbols would be the exact copy of one of the other two. This results in more than one OFDM symbol synchronization overhead per synchronization burst. For the so-called ALOHA environment, Warner [41] proposed the employment of a power detector and subsequent correlation-based detection of a set of received synchronization subcarriers embedded in the data symbols. The received synchronization tones are extracted from the received time-domain signal using an iterative algorithm for updating the synchronization tone values once per sampling interval. For a more detailed discussion on these techniques, the interested reader is referred to the literature [20], [41].

\section{B. Fine Symbol Tracking Overview}

Fine symbol tracking algorithms are generally based on correlation operations either in the time or in the frequency domain. Warner [41] and Bingham [92] employed frequency-domain correlation of the received synchronization pilot tones with known synchronization sequences, while de Couasnon [93] utilized the redundancy of the cyclic prefix by integrating over the magnitude of the difference between the data and the cyclic extension samples. Sandell [46] proposed to exploit the autocorrelation properties of the received time-domain samples imposed by the cyclic extension for fine time-domain tracking.

\section{Frequency Acquisition Overview}

The frequency acquisition algorithm has to provide an initial frequency error estimate, which is sufficiently accurate for the subsequent frequency tracking algorithm to operate reliably. Generally, the initial estimate must be accurate to half a subcarrier spacing. Sari [42] proposed the use of a pilot tone embedded into the data symbol, surrounded by zerovalued virtual subcarriers, so that the frequency-shifted pilot can be located easily by the receiver. Moose [43] suggested a shortened repeated OFDM symbol pair, analogous to his frequency tracking algorithm to be highlighted in the next section. By using a shorter DFT for this reference symbol pair, the subcarrier distance is increased, and thus the frequency error estimation range is extended. Claßen [20], [21] proposed to use binary pseudonoise (PN) or so-called CAZAC training sequences carried by synchronization subcarriers, which are also employed for the frequency tracking. The frequency acquisition, however, is performed by a search for the training sequence in the frequency domain. This is achieved by means of frequency-domain correlation of the received symbol with the training sequence.

\section{Frequency Tracking Overview}

Frequency tracking generally relies on an already established coarse frequency estimation having a frequency error of less than half a subcarrier spacing. Moose [43] suggested the use of the phase difference between subcarriers of repeated OFDM symbols in order to estimate frequency deviations of up to one-half of the subcarrier spacing, while Claßen [20] employed frequency-domain synchronization subcarriers embedded into the data symbols, for which the phase shift between consecutive OFDM symbols can be measured. Daffara [45] and Sandell [46] used the phase of the received signal's autocorrelation function, which represents a phase shift between the received data samples and their repeated copies in the cyclic extension of the OFDM symbols.

\section{E. The Effects of Oscillator Phase Noise}

In practice, a carrier recovery loop has to be employed, in order to synchronize the local oscillator with the remote oscillator and once the synchronization loop is locked, there is no carrier frequency offset. However, the synchronization loop is prone to oscillator phase noise or phase jitter. 


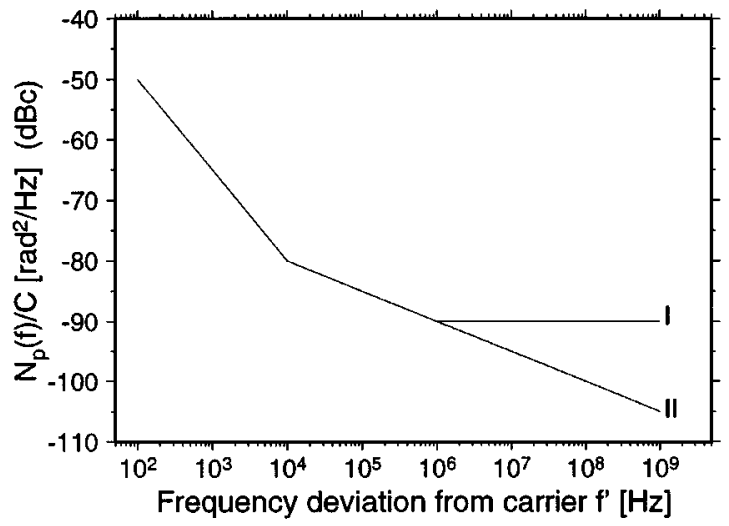

(a)

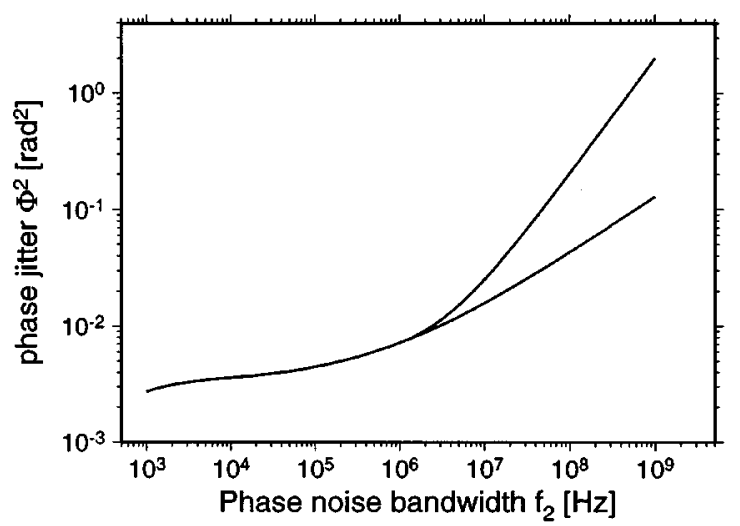

(b)

Fig. 12. Phase noise characterization: (a) spectral phase noise density (phase noise mask) and (b) integrated phase jitter for two different phase noise masks.

This becomes a particularly grave problem in high-frequency, high-bandwidth applications found, for example, in $155-\mathrm{Mb} / \mathrm{s}$ WATM systems operating at $60 \mathrm{GHz}$, such as the applications considered in this contribution. The $60-\mathrm{GHz}$ band is attractive in terms of having a relatively high propagation pathloss due to vapor attenuation and the phenomenon of oxygen-absorption and hence it conveniently curtails cochannel interferences [2]. Furthermore, there is sufficient spectrum available for the $200-\mathrm{MHz}$ bandwidth required by our $155-\mathrm{Mb} / \mathrm{s}$ WATM system. However, at this extremely high frequency, there is a paucity of high-quality oscillators, since no standard systems operate in this frequency band at the time of writing. Hence in this section, we consider briefly the issue of phase noise.

The presence of phase noise is an important limiting factor for an OFDM system's performance [36], [37], [94] and depends on the quality and the operating conditions of the system's radio-frequency hardware. In conventional mobile radio systems around a carrier frequency of $2 \mathrm{GHz}$, the phase noise constitutes typically no severe limitation, however, in the $60-\mathrm{GHz}$ carrier frequency, 225-MHz bandwidth WATM system considered here, its effects were less negligible and hence had to be investigated in more depth. Oscillator noise stems from oscillator inaccuracies in both the transmitter and receiver and manifests itself in the baseband as additional phase and amplitude modulation of the received samples [95]. The oscillator noise influence on the signal depends on the noise characteristics of the oscillators in the system and on the signal bandwidth. It is generally split in amplitude noise $A(t)$ and phase noise $\Phi(t)$, and the influence of the amplitude noise $A(t)$ on the data samples is often neglected. The time-domain functions $A(t)$ and $\Phi(t)$ have Gaussian histograms, and their time-domain correlation is determined by their respective long-term power spectra through the Wiener-Khintchine theorem.

If the amplitude noise is neglected, imperfect oscillators are characterized by the long-term power spectral density (PSD) $N_{p}\left(f^{\prime}\right)$ of the oscillator output signal's phase noise, which is also referred to as the phase noise mask. The variable $f^{\prime}$ represents the frequency distance from the oscillator's nominal carrier frequency in a bandpass model, or, equivalently, the absolute frequency in the baseband. An example of this phase noise mask for a practical oscillator is given in Fig. 12(a). If the phase noise PSD $N_{p}\left(f^{\prime}\right)$ of a specific oscillator is known, then the variance of the phase error $\Phi(t)$ for noise components in a frequency band $\left[f_{1}, f_{2}\right]$ is the integral of the phase noise spectral density over this frequency band as in [95]

$$
\bar{\Phi}^{2}=\int_{f_{1}}^{f_{2}}\left(\frac{2 N_{p}\left(f^{\prime}\right)}{C}\right) d f^{\prime}
$$

where $C$ is the carrier power and the factor two represents the double-sided spectrum of the phase noise. The phase noise variance $\bar{\Phi}^{2}$ is also referred to as the integrated phase jitter, which is depicted in Fig. 12(b).

The phase noise contribution of both the transmitter and receiver can be viewed as an additional multiplicative effect of the radio channel, like fast and slow fading. The performance of the carrier recovery is affected by the phase noise, which in turn degrades the performance of a coherently detected scheme.

For OFDM schemes, multiplication of the received timedomain signal with a time-varying channel transfer function is equivalent to convolving the frequency-domain spectrum of the OFDM signal with the frequency-domain channel transfer function. Since the phase noise spectrum's bandwidth is wider than the subcarrier spacing, this results in energy spillage into other subchannels and therefore in intersubcarrier interference, an effect that will be quantified below. Let us now consider the phase noise model employed in our performance study.

1) Colored Phase Noise Model: The integral $\bar{\Phi}^{2}$ of (21) characterizes the long-term statistical properties of the oscillator's phase and frequency errors due to phase noise. In order to create a time-domain function satisfying the standard deviation $\bar{\Phi}^{2}$, a white Gaussian noise spectrum was filtered with the phase noise mask $N_{p}\left(f^{\prime}\right)$ depicted in Fig. 12(a), which was transformed into the time domain. A frequency resolution of about $50 \mathrm{~Hz}$ was assumed in order to model the shape of the phase noise mask at low frequencies, which led to an FFT transform length of $2^{22}=4194304$ samples for the frequency range of Fig. 12(a). 
Table 2

Two-Sided Phase Noise Mask Used for Simulations: $f^{\prime}$-Frequency Distance from Carrier, $N_{p} / C-$ Normalized Phase Noise Density

\begin{tabular}{c|ccccc}
$f^{\prime}[\mathrm{Hz}]$ & 100 & $1 \mathrm{k}$ & $10 \mathrm{k}$ & $100 \mathrm{k}$ & $1 \mathrm{M}$ \\
\hline$N_{p} / C[\mathrm{~dB}]$ & -50 & -65 & -80 & -85 & -90
\end{tabular}

The resulting time-domain phase noise channel data are a stream of phase error samples, which were used to distort the incoming signal at the receiver. The double-sided phase noise mask used for the simulations is given in Table 2 .

Between the points given in Table 2, a log-linear interpolation is assumed, as shown in Fig. 12(a). As the commercial oscillator's phase noise mask used in our investigations was not specified for frequencies beyond $1 \mathrm{MHz}$, two different cases were considered for frequencies beyond $1 \mathrm{MHz}$ : 1) a phase noise floor at $-90 \mathrm{~dB}$ and 2) a $f^{-1 / 2}$ law. Both of these extended phase noise masks are shown in Fig. 12(a). The integrated phase jitter has been calculated using (21) for both scenarios, and the value of the integral for different noise bandwidths is depicted in Fig. 12(b).

For the investigated 155-Mbits/s WATM system's [76]-[79] double-sided bandwidth of $225 \mathrm{MHz}$, the integration of the phase noise masks results in phase jitter values of $\bar{\Phi}^{2}=0.2303 \mathrm{rad}^{2}$ and $\bar{\Phi}^{2}=0.04533 \mathrm{rad}^{2}$ for the phase noise mask with and without noise floor, respectively.

The simulated BER performance of a 512-subcarrier OFDM system with a subcarrier distance $\Delta f=440 \mathrm{kHz}$ over the two different phase noise channels is depicted in Fig. 13 for coherently detected BPSK and QPSK. In addition to the BER graphs corresponding to the colored phase noise channels described above, graphs of the modems' BER performance over white phase noise channels with the equivalent integrated phase jitter values was also plotted in the figures. It can be observed that the BER performance for both modulation schemes and for both phase noise masks is very similar for the colored and the white phase noise models.

The simulated BER results shown in Fig. 13 show virtually indistinguishable performance for the modems in both the colored and the white phase noise channels, when using BPSK. By contrast, a slight BER difference can be observed for QPSK between the Type 1 and Type 2 phase noise masks, where the corresponding white phase noise results in a better performance than the colored noise. This difference can be explained with the interference's being caused by fewer dominant interfering subcarriers compared to the white phase noise scenario, resulting in a non-Gaussian error histogram.

In summary, phase noise, like all time-varying channel conditions experienced by the time-domain signal, results in intersubcarrier interference in OFDM transmissions. If the bandwidth of the phase noise is high compared to the OFDM subcarrier spacing, then this interference is caused by a high number of contributions from different subcarriers, resulting in a Gaussian noise-like interference. In addition to this noise inflicted upon the received symbols, the signal level in the subcarriers drops by the amount of energy spread over the adjacent subcarriers. The integral over the phase noise mask, termed as phase jitter, is a measure of the signal-to-interference ratio that can be expected in the received subcarriers, if the phase noise has a wide bandwidth and is predominantly white. For narrow-band phase noise, this estimation is pessimistic.

Following the above overview of the associated synchronization issues, we will investigate two different synchronization algorithms, both making use of a reference symbol marking the beginning of a new time frame. This limits the use of both algorithms to systems whose channel access scheme is based on TDMA frames.

\section{F. BER Performance with Frequency Synchronization}

Here we refrain from characterizing the performance of all the previously reviewed synchronization algorithms and refer the interested reader to [1] for implementation-specific details. However, as a representative example, Fig. 14(a) depicts the BER versus channel SNR for BPSK, QPSK, and 16-QAM in an AWGN channel with a frequency error of $\delta f=0.3 \Delta f$. The white symbols in the graph portray the BER performance of an OFDM modem employing no frequency synchronization. It can be seen that the uncorrected frequency errors result in heavy intersubcarrier interference, which manifests itself as a high residual BER of about 5\% for BPSK and QPSK and about $20 \%$ for 16-QAM. The lines in the graph characterize the performance of the modem in the absence of frequency errors. The black markers correspond to the BER recorded with the frequency synchronization algorithm in operation. It can be seen that the performance of the modem employing the frequency synchronization algorithm is nearly indistinguishable from the perfectly synchronized case. In Fig. 14(b), the modem's BER curves for an AWGN channel at a frequency error of $7.5 \Delta f$ are depicted. Since the synchronization algorithm's accuracy does not vary with varying frequency errors, the modem's BER performance employing the synchronization algorithm considered at $\delta f=7.5 \Delta f$ is the same as at $\delta f=0.3 \Delta f$. The BER for the nonsynchronized modem is, however, $50 \%$ and the corresponding markers are off the graph.

The synchronized modem's BER performance in wide-band channels is characterized in Fig. 14(c) and (d). The impulse response used was the WATM impulse response of Fig. 3(a). Perfect knowledge of the CIR was assumed for perfect phase and amplitude correction of the data symbols with coherent detection. Again, the BER curves for both the nonfading and the fading channels show a remarkable correspondence between the ideal performance lines and the performance of the synchronized modems. The modem's performance was unaffected by the estimation accuracy of the time-domain reference symbol synchronization algorithm in all the investigated environments.

In summary, the effects of frequency and timing errors in OFDM transmissions have been characterized. While 


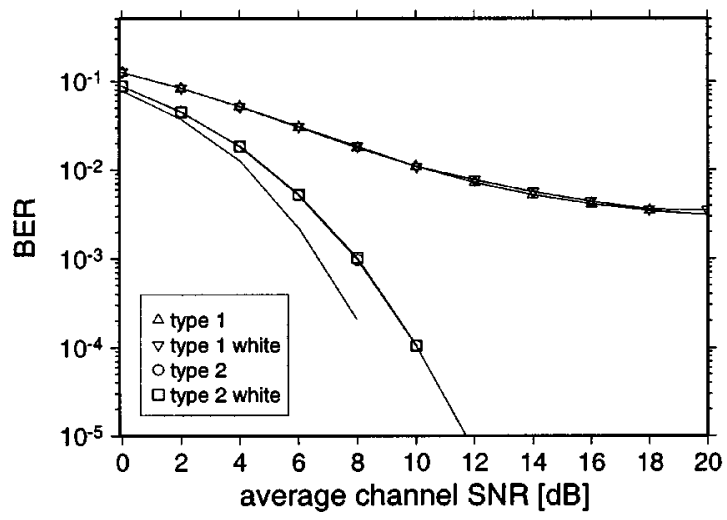

(a)

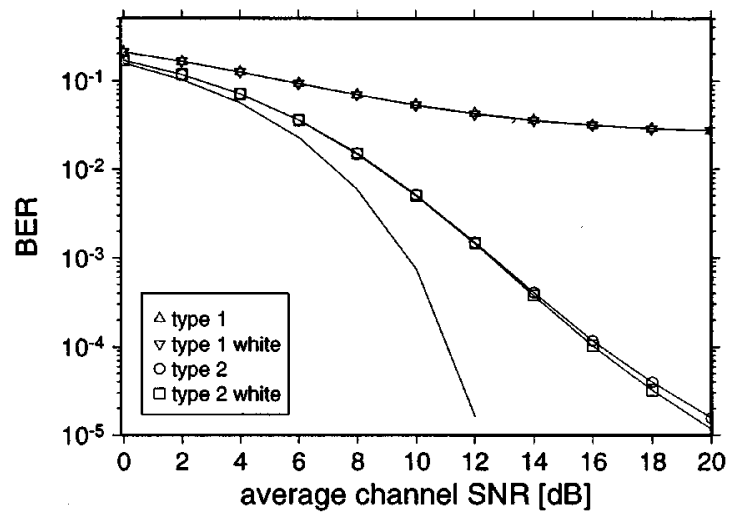

(b)

Fig. 13. BER versus channel SNR for a 512-subcarrier OFDM modem in the presence of phase noise. Type 1 represents the colored phase noise channel with the phase noise mask depicted in Fig. 12(a) assuming a noise floor of $90 \mathrm{rad}^{2} / \mathrm{Hz}$, while Type 2 is the channel without phase noise floor. The curves designated "white" are the corresponding white phase noise results. The lines without markers give the corresponding results in the absence of phase noise.

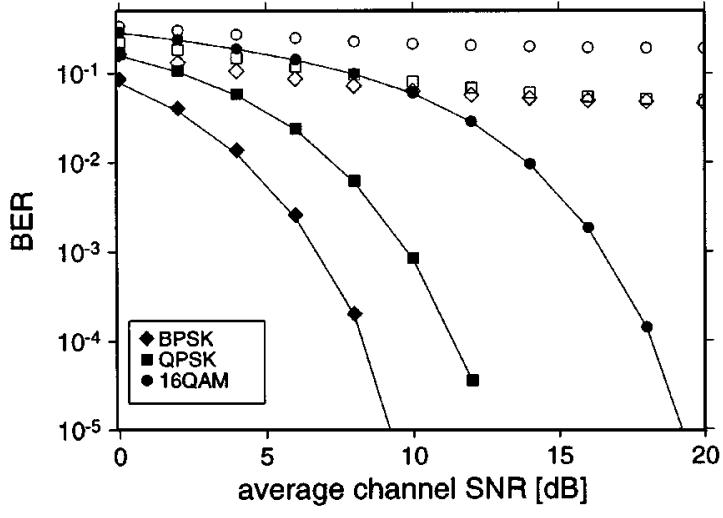

(a)

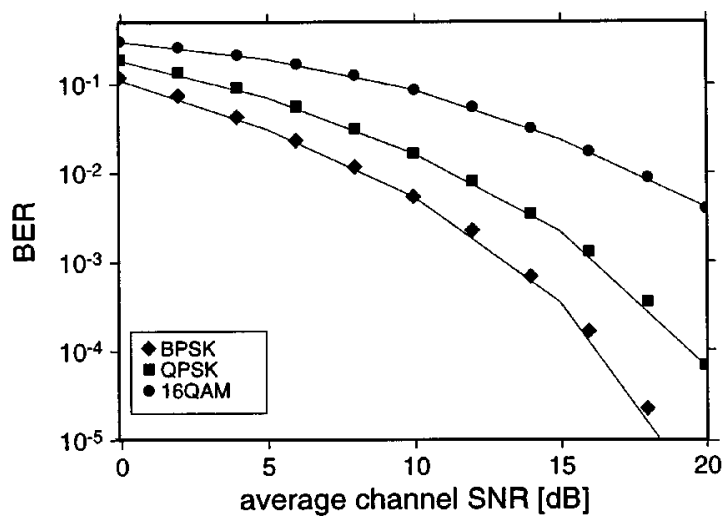

(c)

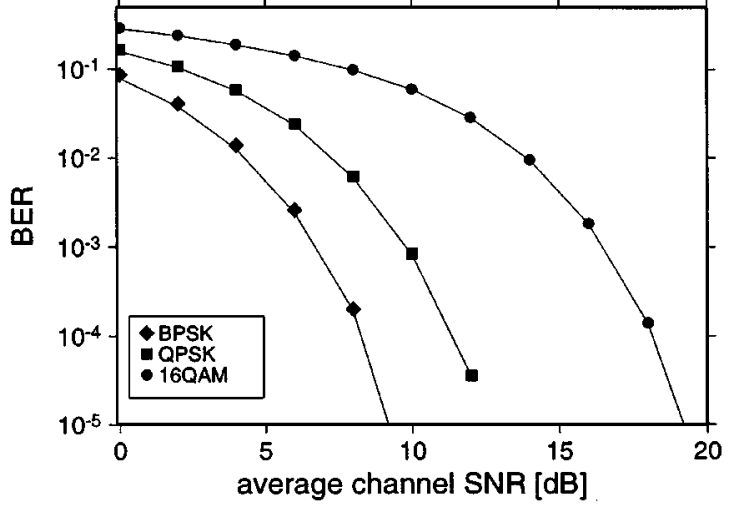

(b)

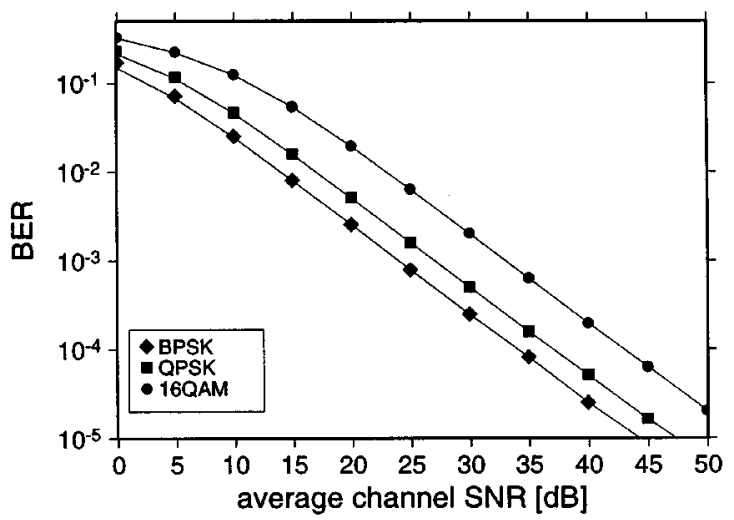

(d)

Fig. 14. BER versus channel SNR performance curves for the 512 subcarrier OFDM system in the presence of fixed frequency errors. The lines indicate the performance for perfectly corrected frequency error, and the white symbols show the performance for uncorrected frequency errors. The black symbols indicate simulations based on frequency error estimation using the time-domain correlation technique. The WATM CIR is shown in Fig. 3(a).

frequency errors result in frequency-domain intersubcarrier interference, timing errors lead to time-domain inter-OFDM symbol interference and to frequency-domain phase rotations. In order to overcome the effects of moderate timing errors, a cyclic postamble and the use of pilot-symbol assisted modulation or differential detection were recommended. Different frequency and timing error estimation algorithms were reviewed and characterized. Let us now in the next section consider the recent advances in the field of sophisticated adaptive OFDM schemes.

\section{ADAPTIVE OFDM}

\section{A. Survey and Motivation}

Steele and Webb [97] proposed adaptive modulation for exploiting the time-variant Shannonian channel capacity of 


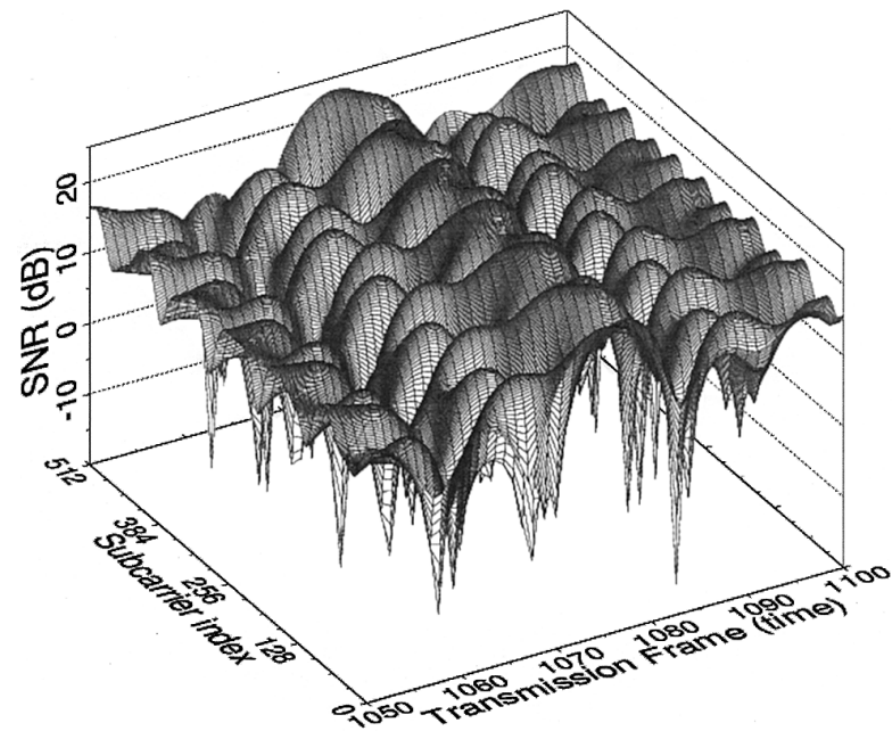

Fig. 15. Instantaneous channel SNR for all 512 subcarriers versus time, for an average channel SNR of $16 \mathrm{~dB}$ over the channel characterized by the impulse response of Fig. 3 .

fading narrow-band channels, which stimulated further research by Sampei et al. [98], Goldsmith et al. [99], Pearce et al.[100], Lau and McLeod [101], and Torrance et al. [102], [103]. The associated principles can also be invoked in the context of parallel modems, as has been demonstrated by Kalet [17], Czylwik et al. [104], and by Chow et al. [105].

Based on the philosophy of the above contributions, below we summarize the ideas behind adaptive modulation. We have seen in Section III that the bit-error probability of different OFDM subcarriers transmitted in time-dispersive channels depends on the frequency-domain channel transfer function. The occurrence of bit errors is normally concentrated in a set of severely faded subcarriers, while in the rest of the OFDM spectrum, often no bit errors are observed. If the subcarriers that will exhibit high bit-error probabilities in the OFDM symbol to be transmitted can be identified and excluded from data transmission, the overall BER can be improved in exchange for a slight loss of system throughput. Since the frequency-domain fading deteriorates the SNR of certain subcarriers, but improves others' above the average SNR value, the potential loss of throughput due to the exclusion of faded subcarriers can be mitigated by employing higher order modulation modes on the subcarriers exhibiting high SNR values.

As a further conceptual augmentation of the above ideas, let us consider the following example. The associated channel SNR of an adaptive OFDM modem is shown in a three-dimensional form in Fig. 15, which was generated with the aid of the FFT of the Rayleigh-faded CIR of Fig. 3. Observe that the instantaneous channel SNR is a function of both time and frequency. An example of the associated timeand frequency-dependent modulation scheme allocation for an adaptive OFDM modem carrying 578 data bits per OFDM symbol at an average channel SNR of $5 \mathrm{~dB}$ is given in Fig. 16(a) for 100 consecutive OFDM symbols. The unused subbands with indexes 15 and 16 contain the virtual

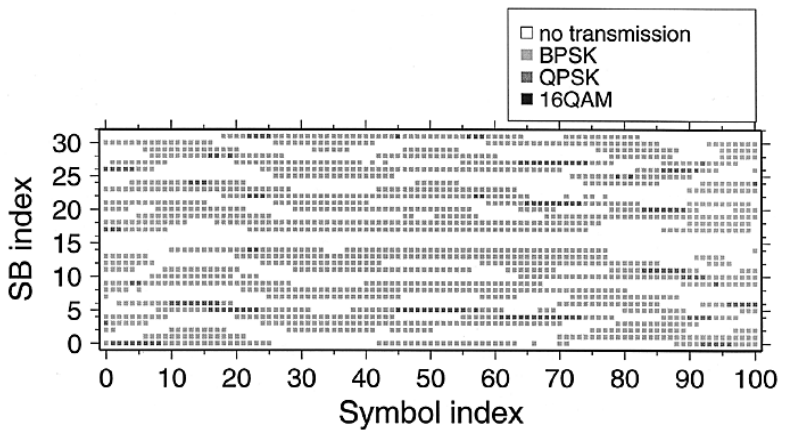

(a)

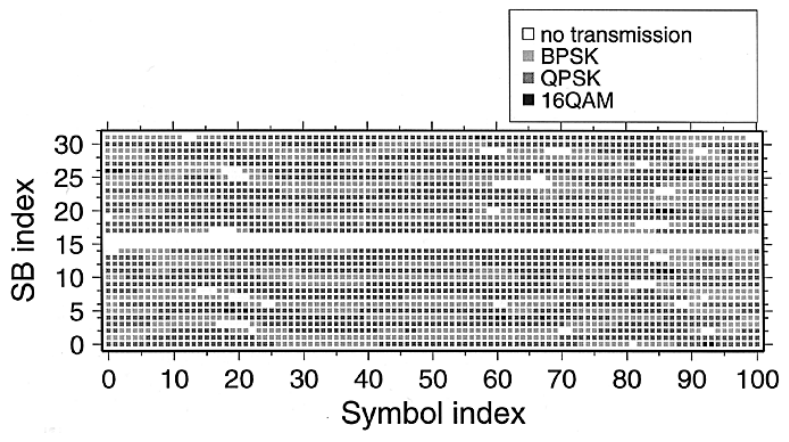

(b)

Fig. 16. An example of modem mode allocation for a 578- and 1458-bit fixed-throughput adaptive OFDM modem over fading time-dispersive channels at 5-dB average channel SNR.

carriers, and therefore do not transmit any useful data. It can be seen that the adaptation algorithm allocates data to the better quality subcarriers on a symbol-by-symbol basis, while keeping the total number of bits per OFDM symbol constant. As a comparison, Fig. 16(b) shows the equivalent overview of the modulation schemes employed for the substantially higher-nearly tripled-fixed bit rate of 1458 bits per OFDM symbol. It can be seen that in order to achieve the throughput target, hardly any subbands are in "no transmission" mode, and overall predominantly higher order modulation schemes, such as QPSK and 16-QAM, have to be employed.

In addition to excluding sets of faded subcarriers and varying the modulation modes employed, other parameters such as the coding rate of error correction coding schemes can also be adapted at the transmitter according to the perceived channel transfer function.

Adaptation of the transmission parameters may be based on the transmitter's perception of the channel conditions in the forthcoming TDMA/time-division duplex (TDD) duplex timeslot. Clearly, this estimation of future channel parameters can only be obtained by extrapolation of previous channel estimations, which are acquired upon detecting each received OFDM symbol. The channel characteristics therefore have to be varying sufficiently slowly compared to the channel estimation interval.

Adapting the transmission technique to the channel conditions on a timeslot-by-timeslot basis for serial modems in narrow-band fading channels has been shown to considerably improve the BER performance [106] for TDD systems assuming duplex reciprocal channels. However, the Doppler 
fading rate of the narrow-band channel has a strong effect on the achievable system performance. If the fading is rapid, then the prediction of the channel conditions for the next transmit timeslot is inaccurate, and therefore the wrong set of transmission parameters may be chosen. If, however, the channel varies slowly, then the data throughput of the system is varying dramatically over time, and large data buffers are required at the transmitters in order to smoothen the bit rate fluctuation. For time-critical applications, such as interactive speech transmission, the potential delays can become problematic. A given single-carrier adaptive system in narrow-band channels will therefore operate efficiently only in a limited range of channel conditions.

Adaptive OFDM modems channels can ease the problem of slowly time-varying channels, since the variation of the signal quality can be exploited in both the time and the frequency domain. The channel conditions still have to be monitored based on the received OFDM symbols, and relatively slowly varying channels have to be assumed, since we have seen in Section III-C2 that OFDM transmissions are not well suited to rapidly varying channel conditions.

\section{B. Adaptive Techniques}

Adaptive modulation is only suitable for duplex communication between two stations, since the transmission parameters have to be adapted using some form of two-way transmission in order to allow channel measurements and signaling to take place. These issues are studied below.

Transmission parameter adaptation is a response of the transmitter to time-varying channel conditions. In order to efficiently react to the changes in channel quality, the following steps have to be taken.

- Channel quality estimation: In order to appropriately select the transmission parameters to be employed for the next transmission, a reliable estimation of the channel transfer function during the next active transmit timeslot is necessary.

- Choice of the appropriate parameters for the next transmission: Based on the prediction of the channel conditions for the next timeslot, the transmitter has to select the appropriate modulation modes for the subcarriers.

- Signaling or blind detection of the employed parameters: The receiver has to be informed, as to which demodulator parameters to employ for the received packet. This information can either be conveyed within the OFDM symbol itself, at the cost of a loss of effective data throughput, or the receiver can attempt to estimate the parameters employed by the remote transmitter by means of blind detection mechanisms. These issues will be made more explicit in the context of Fig. 17.

1) Channel Quality Estimation: The transmitter requires an estimate of the expected channel conditions for the time instant, when the next OFDM symbol is to be transmitted. Since this knowledge can only be gained by prediction from past channel quality estimations, the adaptive system can

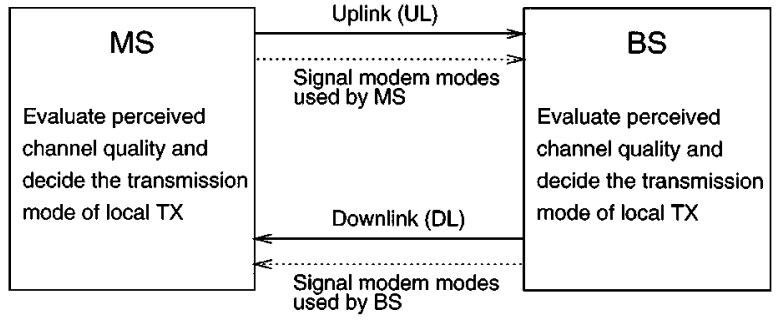

(a)

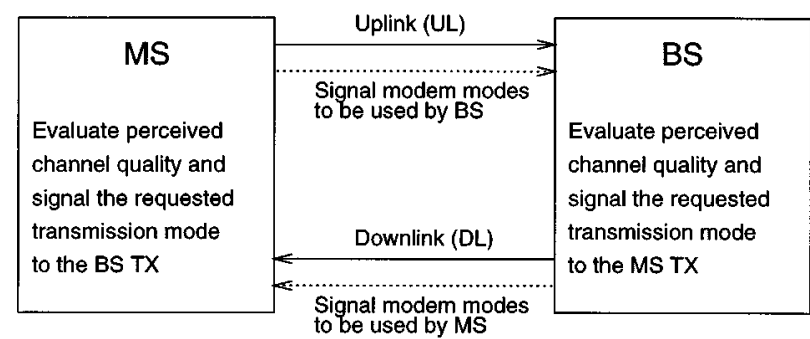

(b)

Fig. 17. Signaling scenarios in adaptive modems.

only operate efficiently in an environment exhibiting relatively slowly varying channel conditions.

The channel quality estimation can be acquired from a range of different sources. If the communication between the two stations is bidirectional and the channel can be considered reciprocal, then each station can estimate the channel quality on the basis of the received OFDM symbols and adapt the parameters of the local transmitter to this estimation. We may refer to such a regime as open-loop adaptation, since there is no feedback between the receiver of a given OFDM symbol and the choice of the modulation parameters. An indoor TDD system in the absence of interference is an example of such a system, and hence a TDD regime is assumed for generating the performance results below. Channel reciprocity issues were addressed, for example, in [107], [108].

If the channel is not reciprocal, as far as the up- and down-link are concerned, as in a frequency-division duplex (FDD) system, then the stations cannot determine the parameters for the next OFDM symbol's transmission from the received symbols. In this case, the receiver has to estimate the channel quality and explicitly signal this perceived channel quality information to the transmitter in the reverse link. Since in this case the receiver explicitly instructs the remote transmitter as to which modem modes to invoke, this regime is referred to as closed-loop adaptation. The adaptation algorithms can-with the aid of this technique-take into account effects such as interference as well as nonreciprocal channels. If the communication between the stations is essentially unidirectional, then a low-rate signaling channel must be implemented from the receiver to the transmitter. If such a channel exists, then the same technique as for nonreciprocal channels can be employed.

Different techniques can be employed to estimate the channel quality. For OFDM modems, the bit-error probability in each subcarrier is determined by the fluctuations of the channel's current frequency-domain channel transfer function $H_{n}$ with the aid of the channel transfer function 
estimates provided by the pilot symbols, provided that no interference is present. The estimate of the channel transfer function $\hat{H}_{n}$ can be acquired by means of pilot-tone based channel estimation. More accurate measures of the channel transfer function can be gained by means of decision-directed or time-domain training sequence based techniques. The estimate of the channel transfer function $\hat{H}_{n}$ does not take into account effects, such as cochannel or intersubcarrier interference. Alternative channel quality measures including interference effects can be devised on the basis of the error correction decoder's soft output information or by means of decision-feedback local SNR estimations.

The delay between the channel quality estimation and the actual transmission of the OFDM symbol in relation to the maximal Doppler frequency of the channel is crucial to the adaptive system's performance. If the channel estimate is obsolete at the time of transmission, then poor system performance will result. For a closed-loop adaptive system, the delays between channel estimation and transmission of the packet are generally longer than for an open-loop adaptive system, and therefore the Doppler frequency of the channel is a more critical parameter for the system's performance than in open-loop adaptive systems.

2) Parameter Adaptation: Different transmission parameters - such as the modulation and coding modes - can be adapted to the anticipated channel conditions. Adapting the number of modulation levels in response to the anticipated local SNR encountered in each subcarrier can be employed, in order to achieve a wide range of different tradeoffs between the received data integrity and throughput. Corrupted subcarriers can be excluded from data transmission and left blank or used, for example, for Crest-factor reduction. A range of different algorithms for selecting the appropriate modulation modes have been proposed in the literature [109], [110].

The adaptive channel coding parameters include code rate, adaptive interleaving and puncturing for convolutional and turbo codes, or varying block lengths for block codes [1]. These techniques can be combined with adaptive modulation mode selection.

Based on the estimated frequency-domain channel transfer function, spectral preequalization at the transmitter of one or both communicating stations can be invoked, in order to partially or fully counteract the frequency-selective fading of the time-dispersive channel. Unlike frequency-domain equalization at the receiver-which corrects for the amplitude and phase errors inflicted upon the subcarriers by the channel-spectral preequalization at the OFDM transmitter can deliver near-constant signal-to-noise levels for all subcarriers. Hence the above concept can be interpreted as power control on a subcarrier-by-subcarrier basis.

In addition to improving the system's BER performance in time-dispersive channels, spectral preequalization can be employed in order to perform all channel estimation and equalization functions at only one of the two communicating duplex stations. Low-cost, low-power consumption mobile stations can communicate with a base station that performs the channel estimation and frequency-domain equalization of the up-link, and uses the estimated channel transfer function for preequalizing the down-link OFDM symbol. This setup would lead to different overall channel quality on the up-link and down-link, and the superior down-link channel quality could be exploited by using a computationally less complex channel decoder having weaker error correction capabilities in the mobile station than in the base station.

If the channel's frequency-domain transfer function is to be fully counteracted by the spectral preequalization upon adapting the subcarrier power to the inverse of the channel transfer function, then the output power of the transmitter can become excessive, if heavily faded subcarriers are present in the system's frequency range. In order to limit the transmitter's maximal output power, hybrid channel preequalization and adaptive modulation schemes can be devised, which would deactivate transmission in deeply faded subchannels, while retaining the benefits of preequalization in the remaining subcarriers.

3) Signaling the Parameters: Signaling plays an important role in adaptive systems, and the range of signaling options is summarized in Fig. 17 for both open-loop and closed-loop signaling, as well as for blind detection. If the channel quality estimation and parameter adaptation have been performed at the transmitter of a particular link, based on open-loop adaptation, then the resulting set of parameters has to be communicated to the receiver in order to successfully demodulate and decode the OFDM symbol. If the receiver itself determines the requested parameter set to be used by the remote transmitter-the closed-loop scenario - then the same amount of information has to be transported to the remote transmitter in the reverse link. If this signaling information is corrupted, then the receiver is generally unable to correctly decode the OFDM symbol corresponding to the incorrect signaling information.

Unlike adaptive serial systems, which employ the same set of parameters for all data symbols in a transmission packet [102], [103], adaptive OFDM systems have to react to the frequency-selective nature of the channel, by adapting the modem parameters across the subcarriers. The resulting signaling overhead could become significantly higher than that for serial modems, and can be prohibitive, for example, for subcarrier-by-subcarrier modulation mode adaptation. In order to overcome these limitations, efficient and reliable signaling techniques have to be employed for practical implementation of adaptive OFDM modems.

If some flexibility in choosing the transmission parameters is sacrificed in an adaptation scheme, like in the subband adaptive OFDM schemes described below [110], [109], then the amount of signaling can be reduced. Alternatively, blind parameter detection schemes can be devised, which require little or no signaling information, respectively. A simple blind modulation scheme detection algorithms will be highlighted in Section VI-D2 [110], [109].

The effects of transmission parameter adaptation for OFDM systems on the overall communication system have to be appraised in at least the following areas: data buffering and latency due to varying data throughput [102], the effects of cochannel interference and bandwidth efficiency [103]. 


\section{Choice of the Modulation Modes}

In [110] and [109], the two communicating stations use the open-loop predicted channel transfer function acquired from the most recent received OFDM symbol, in order to allocate the appropriate modulation modes to the subcarriers. The modulation modes were chosen from the set of BPSK, QPSK, 16-QAM, as well as "No Transmission," for which no signal was transmitted. These modulation modes are denoted by $M_{n}$, where $n \in(0,1,2,4)$ is the number of data bits associated with one data symbol of each mode.

In order to keep the system complexity low, the modulation mode was not varied on a subcarrier-by-subcarrier basis, but instead the total OFDM bandwidth of 512 subcarriers was split into blocks of adjacent subcarriers, referred to as subbands, and the same modulation scheme was employed for all subcarriers of the same subband. This substantially simplified the task of signaling the modem mode and rendered the employment of alternative blind detection mechanisms feasible, which will be discussed in Section VI-D.

Three subband modulation mode allocation algorithms were investigated in the literature [109], [110]: a fixed-threshold controlled algorithm, an upper bound BER estimator and a fixed-throughput adaptation algorithm.

\section{1) Fixed-Threshold Adaptation Algorithm: The} fixed-threshold algorithm was derived from the adaptation algorithm proposed by Torrance for serial modems [111]. In the case of a serial modem, the channel quality was assumed to be constant for all symbols in the timeslot, and hence the channel had to be slowly varying, in order to allow accurate channel quality prediction. Under these circumstances, all data symbols in the transmit time slot employed the same modulation mode, chosen according to the predicted SNR. The SNR thresholds for a given long-term target BER were determined by Powell optimization [111]. Torrance assumed two uncoded target BER's: $1 \%$ for a high-data-rate "speech" system, and $10^{-4}$ for a higher integrity, lower data-rate "data" system. The resulting SNR thresholds $l_{n}$ invoked for activating a given modulation mode $M_{n}$ in a slowly Rayleigh fading narrow-band channel for both systems are given in Table 3. Specifically, the modulation mode $M_{n}$ is selected if the instantaneous channel SNR exceeds the switching level $l_{n}$.

This adaptation algorithm originally assumed a constant instantaneous SNR over all of the transmission burst's symbols, but in the case of an OFDM system in a frequency-selective channel the channel quality varies across the different subcarriers. For subband adaptive OFDM transmission, this implies that if the subband width is wider than the channel's coherence bandwidth [1], then the original switching algorithm cannot be employed. In the performance evaluations, the lowest quality subcarrier in the subband was employed for the adaptation algorithm based on the thresholds given in Table 3. The performance of the 16-subband adaptive system over the WATM Rayleigh fading channel of Fig. 3 is shown in Fig. 18.

Adjacent or consecutive timeslots have been used for the up-link and down-link slots in these simulations, so that
Table 3

Optimized Switching Levels for Adaptive Modulation over Rayleigh Fading Channels for the "Speech" and "Data" System, Shown in Instantaneous Channel SNR dB (from [111])

\begin{tabular}{c|c|c|c|c}
\hline & $l_{0}$ & $l_{1}$ & $l_{2}$ & $l_{4}$ \\
\hline speech system & $-\infty$ & 3.31 & 6.48 & 11.61 \\
\hline data system & $-\infty$ & 7.98 & 10.42 & 16.76 \\
\hline
\end{tabular}

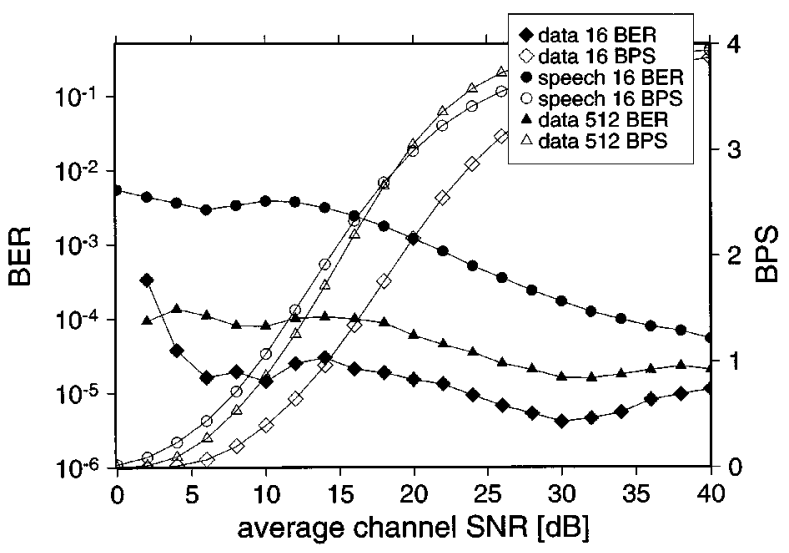

Fig. 18. BER and bit per symbol (BPS) throughput performance of the 16-subband 512-subcarrier switching level adaptive OFDM modem employing BPSK, QPSK, 16-QAM and "no transmission" over the Rayleigh fading time dispersive channel of Fig. 3 using the switching thresholds of Table 3.

the delay between channel estimation and transmission was rendered as short as possible. Fig. 18 shows the long-term average BER and throughput of the studied modem for the "speech" and "data" switching levels of Table 3 as well as for a subcarrier-by-subcarrier adaptive modem employing the "data" switching levels. The results show the typical behavior of a variable-throughput adaptive OFDM system, which constitutes a tradeoff between the best BER and best throughput performance. For low SNR values, the system achieves a low BER by transmitting very few bits and only when the channel conditions allow. With increasing long-term SNR, the throughput increases, without significant change in the BER. For high SNR values, the BER drops as the throughput approaches its maximum of 4 bits per symbol, since the highest order constellation was 16-QAM.

It can be seen from the figure that the adaptive system performs better than its target BER's of $10^{-2}$ and $10^{-4}$ for the "speech" and "data" system, respectively, resulting in measured BER's lower than the targets. This can be explained by the adaptation regime, which was based on the conservative principle of using the lowest quality subcarrier in each subband for channel quality estimation, leading to a pessimistic channel quality estimate for the entire subband. For low values of SNR, the throughput in bits per data symbol is low and exceeds the fixed BPSK throughput of $1 \mathrm{bit} / \mathrm{symbol}$ only for SNR values in excess of 9.5 and $14 \mathrm{~dB}$ for the "speech" and "data" systems, respectively. 

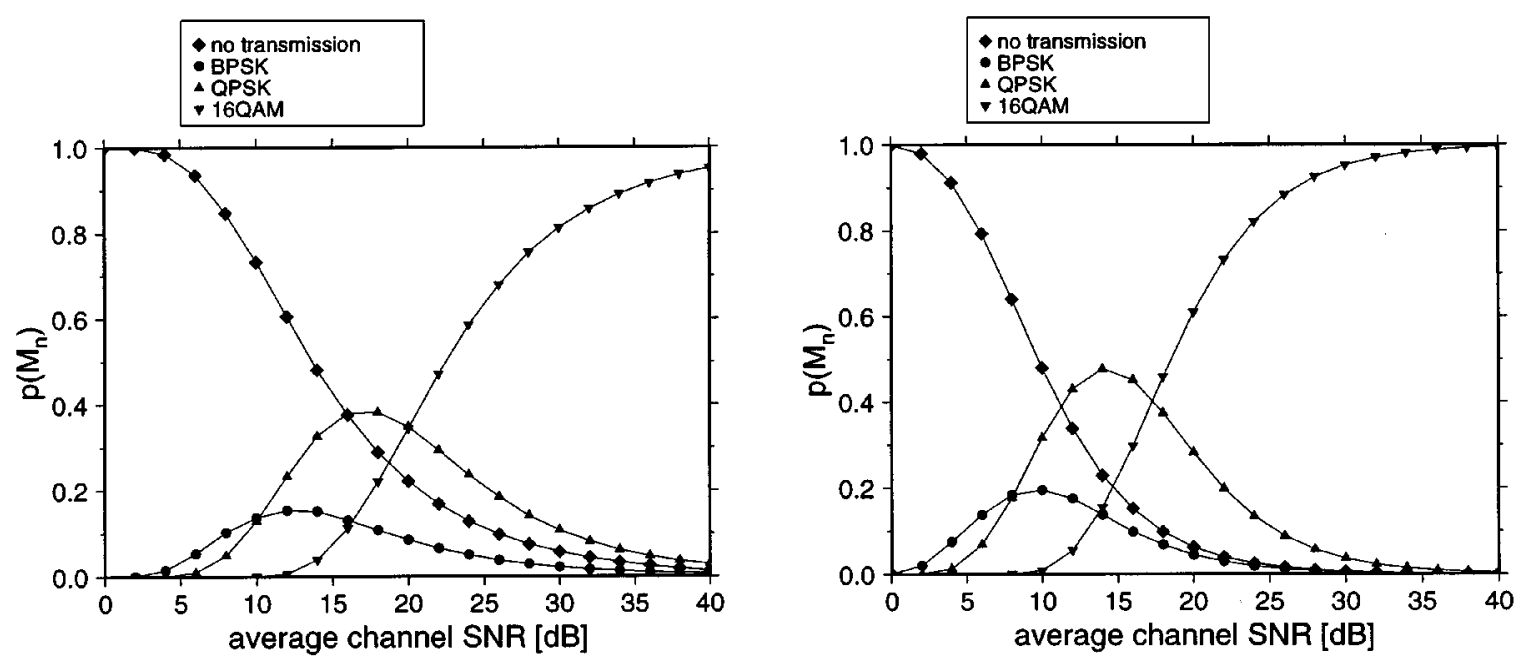

Fig. 19. Histograms of modulation modes versus channel SNR for the "data" switching level adaptive 512-subcarrier 16-subband OFDM modem over the Rayleigh-fading time-dispersive channel of Fig. 3 using the switching thresholds of Table 3.

The upper bound performance of the system with subcarrier-by-subcarrier adaptation is also portrayed in the figure, shown as 512 independent subbands, for the "data" optimized set of threshold values. It can be seen that in this case, the target BER of $10^{-4}$ is closely met over a wide range of SNR values from about 2 to $20 \mathrm{~dB}$, and that the throughput is considerably higher than in the case of the 16-subband modem. This is the result of more accurate subcarrier-bysubcarrier channel quality estimation and fine-grained adaptation, leading to better exploitation of the available channel capacity.

Fig. 19 shows the long-term modulation mode histograms for a range of channel SNR values for the "data" switching levels in both the 16-subband and the subcarrier-by-subcarrier adaptive modems using the switching thresholds of Table 3. Comparison of the graphs shows that higher order modulation modes are used more frequently by the subcarrier-bysubcarrier adaptation algorithm, which is in accordance with the overall throughput performance of the two modems in Fig. 18.

The throughput penalty of employing subband adaptation depends on the frequency-domain variation of the channel transfer function. If the subband bandwidth is lower than the channel's coherence bandwidth, then the assumption of constant channel quality per subband is closely met, and the system performance is equivalent to that of a subcarrier-bysubcarrier adaptive scheme.

2) Subband BER Estimator Adaptation Algorithm: We have seen above that the fixed switching level based algorithm leads to a throughput performance penalty, if used in a subband adaptive OFDM modem, when the channel quality is not constant throughout each subband. This is due to the conservative adaptation based on the subcarrier experiencing the most hostile channel in each subband.

An alternative scheme taking into account the nonconstant SNR values $\gamma_{j}$ across the $N_{s}$ subcarriers in the $j$ th subband can be devised by calculating the expected overall bit-error probability for all available modula-

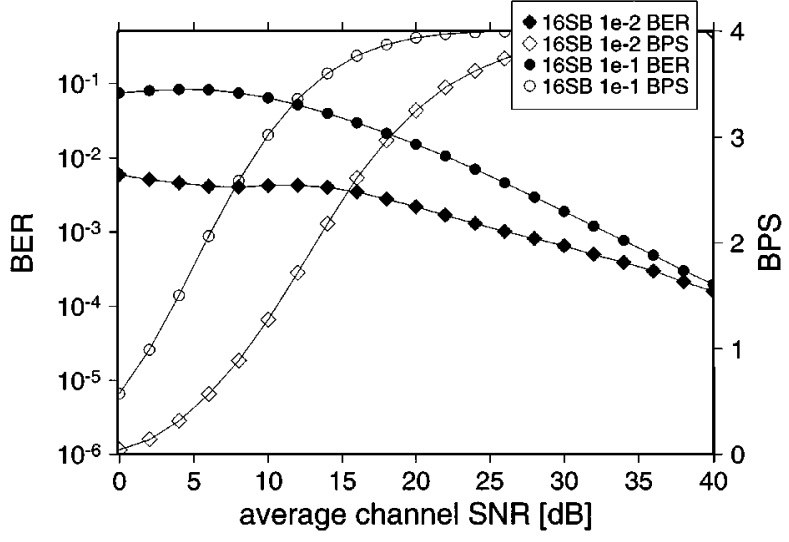

Fig. 20. BER and BPS throughput performance of the 16-subband 512-subcarrier BER estimator adaptive OFDM modem employing BPSK, QPSK, 16-QAM and "no transmission" over the Rayleigh-fading time-dispersive channel of Fig. 3.

tion modes $M_{n}$ in each subband, which is denoted by $\bar{p}_{e}(n)=1 / N_{s} \sum_{j} p_{e}\left(\gamma_{j}, M_{n}\right)$. For each subband, the mode having the highest throughput, whose estimated BER is lower than a given threshold, is then chosen. While the adaptation granularity is still limited to the subband width, the channel quality estimation includes not only the most corrupted subcarrier, which leads to an improved throughput.

Fig. 20 shows the BER and throughput performance for the 16-subband adaptive OFDM modem employing the BER estimator adaptation algorithm in the Rayleigh-fading timedispersive channel of Fig. 3. The two sets of curves in the figure correspond to target BER's of $10^{-2}$ and $10^{-1}$, respectively. Comparing the modem's performance for a target BER of $10^{-2}$ with that of the "speech" modem in Fig. 18, it can be seen that the BER estimator algorithm results in significantly higher throughput, while meeting the BER requirements. The BER estimator algorithm is readily adjustable to different target BER's, which is demonstrated in the figure for a target BER of $10^{-1}$. Such adjustability is beneficial 
when combining adaptive modulation with channel coding, as will be discussed in Section VI-E.

\section{Signaling and Blind Detection}

The adaptive OFDM receiver has to be informed of the modulation modes used for the different subbands. This information can either be conveyed using signaling subcarriers in the OFDM symbol itself, or the receiver can employ blind detection techniques in order to estimate the transmitted symbols' modulation modes, as seen in Fig. 17 [109], [110].

1) Signaling: The simplest way of signaling the modulation mode employed in a subband is to replace one data symbol by an $M$ PSK symbol, where $M$ is the number of possible modulation modes. In this case, reception of each of the constellation points directly signals a particular modulation mode in the current subband. For four modulation modes - and assuming perfect phase recovery-the probability of a signaling error $p_{s}(\gamma)$, when employing one signaling symbol, is the symbol error probability of QPSK. Then the correct subband mode signaling probability is

$$
\left(1-p_{s}(\gamma)\right)=\left(1-p_{b, \mathrm{QPSK}}(\gamma)\right)^{2}
$$

where $p_{b}$, QPSK is the bit-error probability for QPSK

$$
p_{b, \operatorname{QPSK}}(\gamma)=Q(\sqrt{\gamma})=\frac{1}{2} \cdot \operatorname{erfc}\left(\sqrt{\frac{\gamma}{2}}\right)
$$

which leads to the expression for the modulation mode signaling error probability of

$$
p_{s}(\gamma)=1-\left(1-\frac{1}{2} \cdot \operatorname{erfc}\left(\sqrt{\frac{\gamma}{2}}\right)\right)^{2}
$$

The modem mode signaling error probability can be reduced by employing multiple signaling symbols and maximum ratio combining of the received signaling symbols $R_{s, n}$, in order to generate the decision variable $R_{s}^{\prime}$ prior to decision

$$
R_{s}^{\prime}=\sum_{n=1}^{N_{s}} R_{s, n} \cdot \hat{H}_{s, n}
$$

where $N_{s}$ is the number of signaling symbols per subband, the quantities $R_{s, n}$ are the received symbols in the signaling subcarriers and $\hat{H}_{s, n}$ represents the estimated values of the frequency-domain channel transfer function at the signaling subcarriers. Assuming perfect channel estimation and constant values of the channel transfer function across the group of signaling subcarriers, the signaling error probability for $N_{s}$ signaling symbols can be expressed as

$$
p_{s}^{\prime}\left(\gamma, N_{s}\right)=1-\left(1-\frac{1}{2} \cdot \operatorname{erfc}\left(\sqrt{\frac{N_{s} \gamma}{2}}\right)\right)^{2}
$$

Fig. 21 shows the signaling error rate in an AWGN channel for one, two, four, and eight signaling symbols per subband, respectively. It can be seen that doubling the number of signaling subcarriers improves the performance by $3 \mathrm{~dB}$. Modem mode detection error ratios (DER's) below $10^{-5}$ can be achieved at $10-\mathrm{dB}$ SNR over AWGN channels if two signaling symbols are used. The signaling symbols for a given subband can be interleaved across the

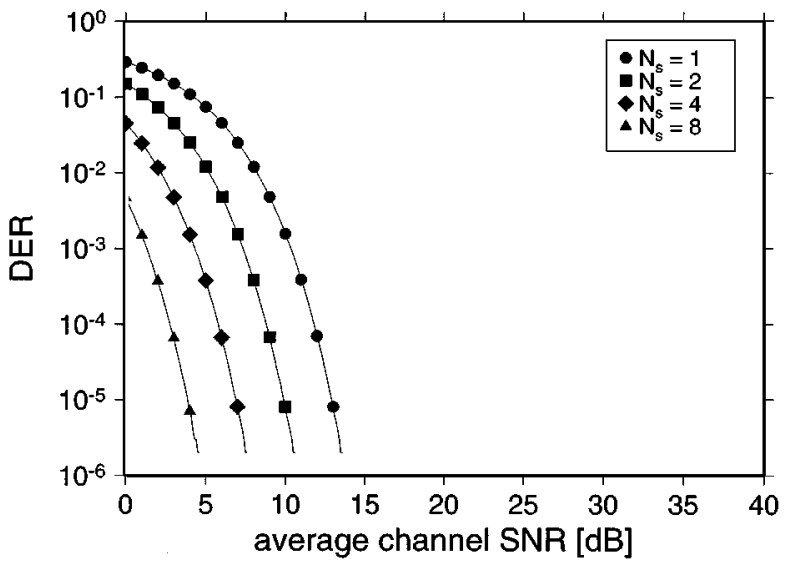

Fig. 21. Modulation mode DER if signaling with maximum ratio combining is employed for QPSK symbols in an AWGN channel for one, two, four, and eight signaling symbols per subband, evaluated from (26).

entire OFDM symbol bandwidth, in order to benefit from frequency diversity in fading wide-band channels.

As seen in Fig. 17, blind detection algorithms aim to estimate the employed modulation mode directly from the received data symbols, therefore avoiding the loss of data capacity due to signaling subcarriers. Two algorithms have been proposed in the literature [109], [110], one based on SNR estimation and another one incorporating error correction coding. Let us briefly highlight the conceptually more simple one in the next section.

2) Blind Detection by SNR Estimation: The receiver has no a priori knowledge of the modulation mode employed in a particular received subband and estimates this parameter by quantising the defaded received data symbols $R_{n} / \hat{H}_{n}$ in the subband to the closest symbol $\hat{R}_{n, m}$ for all possible modulation modes $M_{m}$ for each subcarrier index $n$ in the current subband. The decision-directed error energy $e_{m}$ between the ideal constellation phasor positions and the received phasors is calculated for each modulation mode according to

$$
e_{m}=\sum_{n}\left(R_{n} / \hat{H}_{n}-\hat{R}_{n, m}\right)^{2}
$$

and then the modulation mode $M_{m}$, which minimizes $e_{m}$, is chosen for the demodulation of the subband.

The DER of the blind modulation mode detection algorithm described in this section for a 512-subcarrier OFDM modem in an AWGN channel is depicted in Fig. 22. It can be seen that the detection performance depends on the number of symbols per subband, with fewer subbands and therefore longer symbol sequences per subband leading to a better detection performance. It is apparent, however, that the number of available modulation modes has a more significant effect on the detection reliability than the block length. If all four legitimate modem modes are employed, then reliable detection of the modulation mode is only guaranteed for AWGN SNR values of more than 15-18 dB, depending on the number of subbands per OFDM symbol. If only $M_{0}$ and $M_{1}$ are employed, however, the estimation accuracy is dramatically improved. In this case, AWGN SNR values above 5-7 dB are sufficient to ensure reliable detection. 


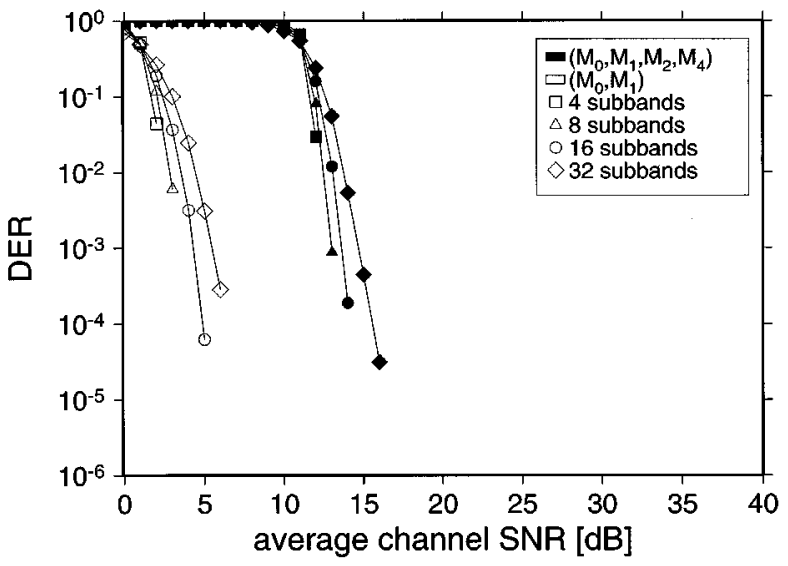

Fig. 22. Blind modulation mode DER for 512-subcarrier OFDM systems employing $\left(M_{0}, M_{1}\right)$ as well as for $\left(M_{0}, M_{1}, M_{2}, M_{4}\right)$ for different numbers of subbands in an AWGN channel.

Fig. 23 shows the BER performance of the fixed-threshold "data"-type 16-subband adaptive system in the fading wide-band channel of Fig. 3 for both sets of modulation modes, namely, for $\left(M_{0}, M_{1}\right)$ and $\left(M_{0}, M_{1}, M_{2}, M_{4}\right)$ with blind modulation mode detection. Erroneous modulation mode decisions were assumed to yield a BER of $50 \%$ in the received block. This is optimistic, since in a realistic scenario the receiver would have no knowledge of the number of bits actually transmitted, leading to loss of synchronization in the data stream. This problem is faced by all systems having a variable throughput and not employing an ideal reliable signaling channel. This impediment must be mitigated by data synchronization measures.

It can be seen from Fig. 23 that while blind modulation mode detection yields poor performance for the quadruple-mode adaptive scheme, the twin-mode scheme exhibits BER results consistently better than $10^{-4}$.

\section{E. Subband Adaptive OFDM and Channel Coding}

Adaptive modulation can reduce the BER to a level where channel decoders can perform well. Fig. 24 shows both the uncoded and coded BER performance of a 512-subcarrier OFDM modem in the fading wide-band channel of Fig. 3, assuming perfect channel estimation. The channel coding employed in this set of experiments was a turbo coder [112] with a data block length of 1000 bits, employing a random interleaver and eight decoder iterations. The log-MAP decoding algorithm was used [113]. The constituent half-rate convolutional encoders were of constraint length three, with octally represented generator polynomials of $(7,5)$ [1]. It can be seen that the turbo decoder provides a considerable coding gain for the different fixed modulation schemes, with a BER of $10^{-4}$ for SNR values of $13.8,17.3$, and $23.2 \mathrm{~dB}$ for BPSK, QPSK, and 16-QAM transmission, respectively.

Fig. 25 depicts the BER and throughput performance of the same decoder employed in conjunction with the adaptive OFDM modem for different adaptation algorithms. Fig. 25(a) shows the performance for the "speech" system employing the switching levels listed in Table 3. As expected, the half-rate channel coding results in a halved throughput compared to the uncoded case but offers low-BER transmission over the channel of Fig. 3 for SNR values down to $0 \mathrm{~dB}$, maintaining a BER below $10^{-6}$.

Further tuning of the adaptation parameters can ensure a better average throughput while retaining error-free data transmission. The switching-level-based adaptation algorithm of Table 3 is difficult to control for arbitrary BER's, since the set of switching levels was determined by an optimization process for uncoded transmission. Since the turbo-codec has a nonlinear BER versus SNR characteristic, direct switching-level optimization is an arduous task. The subband BER predictor of Section VI-C2 is easier to adapt to a channel codec, and Fig. 25(b) shows the performance for the same decoder, with the adaptation algorithm employing the BER-prediction method having an upper BER bound of $1 \%$. It can be seen that the less stringent uncoded BER constraints when compared to Fig. 25(a) lead to a significantly higher throughput for low SNR values. The turbo-decoded data bits are error-free; hence a further increase in throughput is possible while maintaining a high degree of coded data integrity.

The second set of curves in Fig. 25(b) show the system's performance if an uncoded target BER of $10 \%$ is assumed. In this case, the turbo decoder's output BER is below $10^{-5}$ for all the SNR values plotted and shows a slow decrease for increasing values of SNR. The throughput of the system, however, exceeds 0.5 data bits per symbol for SNR values of more than $2 \mathrm{~dB}$. In closing, we note that it is an attractive adaptive OFDM property that the adaptive modulation mode selection results in less bursty error statistics than fixed modulation, which improves the achievable performance of the channel codec.

\section{PREEQUALIZATION}

We have seen above how the receiver's estimate of the channel transfer function can be employed by the transmitter in order to dramatically improve the performance of an OFDM system by adapting the subcarrier modulation modes to the channel conditions. For subchannels exhibiting a low signal-to-noise ratio, robust modulation modes are used, while for subcarriers having a high SNR, high-throughput multilevel modulation modes can be employed. An alternative approach to combating the frequency-selective channel behavior was proposed in [114], applying preequalization to the OFDM symbol prior to transmission on the basis of the anticipated channel transfer function. We will highlight a range of related topics in this section.

\section{A. Motivation}

As discussed above, the received data symbol $R_{n}$ of subcarrier $n$ over a slowly time-varying time-dispersive channel can be characterized by

$$
R_{n}=S_{n} \cdot H_{n}+n_{n}
$$

where

$S_{n} \quad$ transmitted data symbol;

$H_{n} \quad$ channel transfer function of subcarrier $n$;

$n_{n} \quad$ noise sample. 


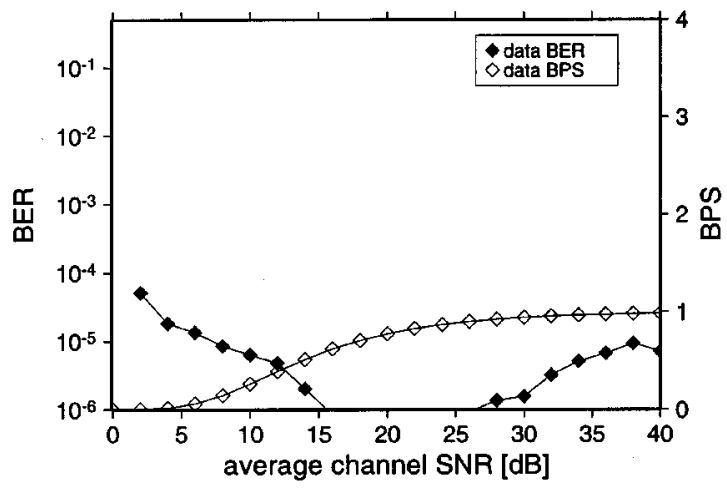

(a)

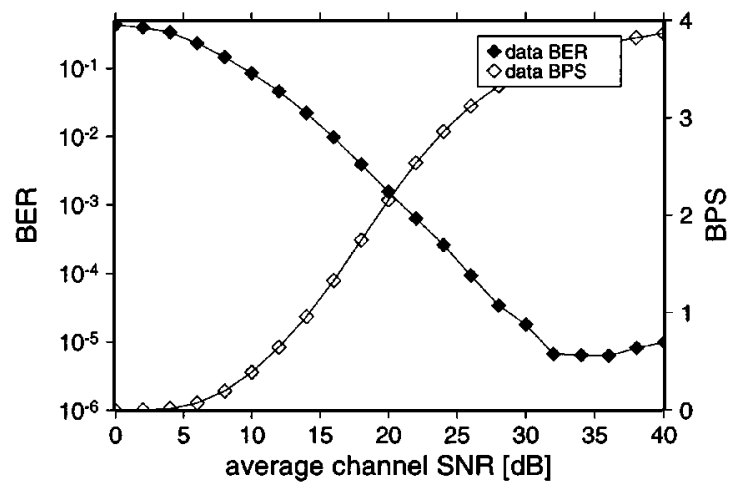

(b)

Fig. 23. BER and BPS throughput performance of a 16-subband 512-subcarrier adaptive OFDM modem employing (a) No Transmission $\left(M_{0}\right)$ and BPSK $\left(M_{1}\right)$ or (b) $\left(M_{0}, M_{1}, M_{2}, M_{4}\right)$, both using the data-type switching levels of Table 3 and the SNR-based blind modulation mode detection of Section VI-D2 over the Rayleigh-fading time-dispersive channel of Fig. 3.

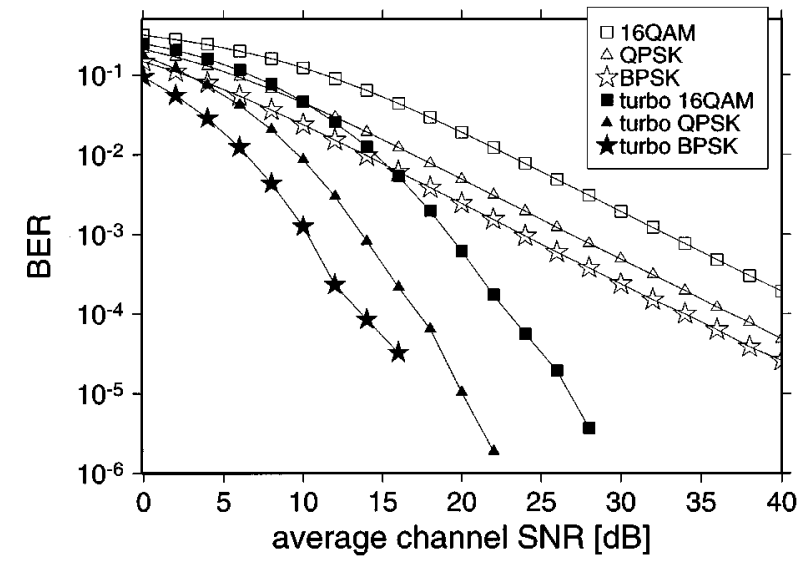

Fig. 24. BER performance of the 512-subcarrier OFDM modem in the fading time-dispersive channel of Fig. 3 for both uncoded and half-rate turbo-coded transmission, using eight-iteration log-MAP turbo decoding, 1000-bit random interleaver, and constraint length of three.

The pilot-based frequency-domain equalization at the receiver-which is necessary for nondifferential detection of the data symbols-corrects the phase and amplitude of the received data symbols using the estimate of the channel transfer function $\hat{H}_{n}$ as follows:

$$
R_{n}^{\prime}=R_{n} / \hat{H}_{n}=S_{n} \cdot H_{n} / \hat{H}_{n}+n_{n} / \hat{H}_{n} .
$$

If the estimate $\hat{H}_{n}$ is accurate, this operation defades the constellation points before decision. However, upon defading, the noise sample $n_{n}$ is amplified by the same amount as the signal, therefore failing to improve the SNR of the received sample.

Preequalization for the OFDM modem operates by scaling the data symbol of subcarrier $n, S_{n}$, by a preequalization function $E_{n}$, computed from the inverse of the anticipated channel transfer function, prior to transmission. At the receiver, no equalization is performed; hence the received symbols can be expressed as

$$
R_{n}=S_{n} \cdot E_{n} \cdot H_{n}+n_{n}
$$

Since no equalization is performed, there is no noise amplification at the receiver. Similarly to the adaptive modulation techniques illustrated above, preequalization is only applicable to a duplex link, since the transmitted signal is adapted to the specific channel conditions perceived by the receiver. As for other adaptive schemes, the transmitter needs an estimate of the current frequency-domain channel transfer function, which can be obtained from the received signal in the reverse link, as seen in Fig. 17.

\section{B. Preequalization with Subband Blocking}

Direct channel inversion at the transmitter is not practical, as the output power fluctuations are prohibitive; excluding those subcarriers that are faded too low can be used to limit the necessary transmit power. Analogously to the adaptive modulation schemes above, the transmitter decides for all subcarriers in each subband whether to transmit data or not. If preequalization is possible under the power constraints, then the subcarriers are modulated with the preequalized data symbols. The information on whether a subband is used for transmission or not is signaled to the receiver.

Since no attempt is made to transmit in the subbands that cannot be preequalized, the power not employed in the blank subcarriers can be used for "boosting" the data-bearing subbands. This scheme allows for a more flexible preequalization algorithm than the fixed-threshold-based method described above, which is summarized as follows.

- Calculate the necessary transmit power $p_{n}$ for each subband $n$, assuming perfect preequalization.

- Sort subbands according to their required transmit power $p_{n}$.

- Select subband $n$ with the lowest power $p_{n}$, and add $p_{n}$ to the total transmit power. Repeat this procedure with the next lowest power, until no further subbands can be added without the total power $\sum p_{j}$ exceeding the power limit $l$.

Fig. 26 depicts the 16-QAM BER performance over the WATM channel of Fig. 3. Three different blocking thresholds, namely, 0, 6 and $12 \mathrm{~dB}$, were used, where subbands requiring more power than the allowable maximum were blocked from transmission. The BER floor stems from the channel variability, due to the time delay between channel 


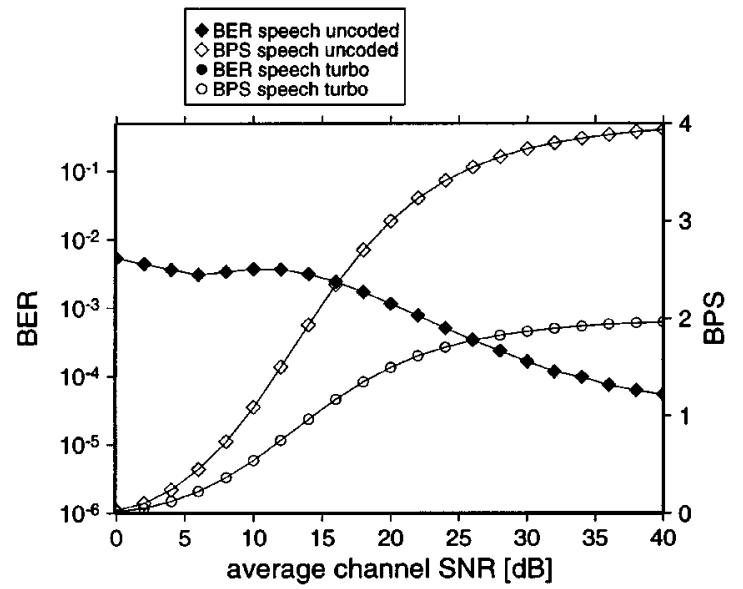

(a)

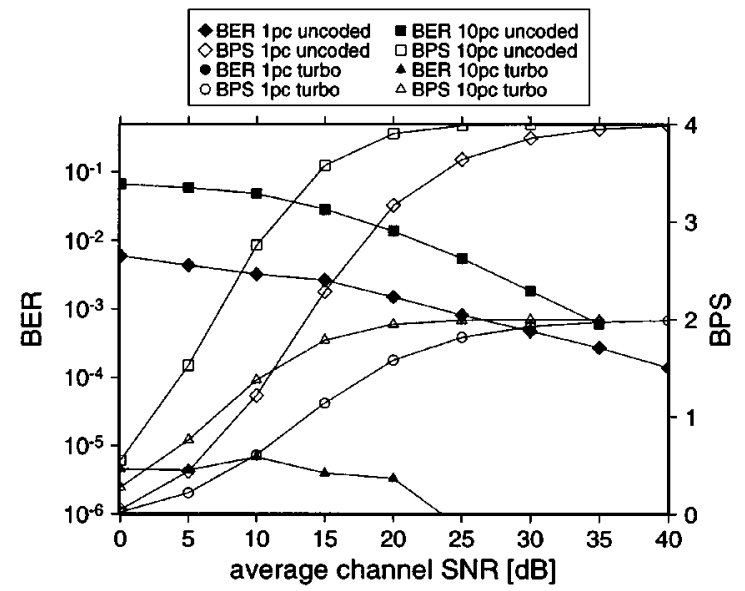

(b)

Fig. 25. BER and BPS throughput performance of 16-subband 512-subcarrier adaptive turbo-coded and uncoded OFDM modem employing $\left(M_{0}, M_{1}, M_{2}, M_{4}\right)$ for (a) speech-type switching levels of Table 3 and (b) a maximal estimated subband BER of $1 \%$ and $10 \%$ over the channel of Fig. 3. The turbo-coded transmission over the speech system and the $1 \%$ maximal BER system are error free for all examined SNR values, and therefore the corresponding BER curves are omitted from the graphs.

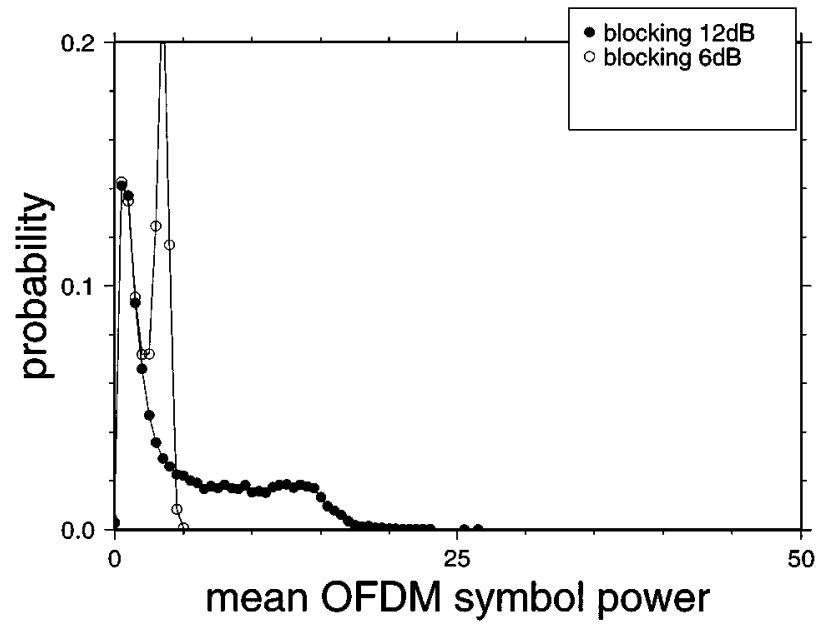

Fig. 26. BER performance of the 512-subcarrier 16-QAM OFDM modem over the fading WATM channel of Fig. 3 employing 16-subband preequalization with blocking and a delay of one timeslot between the instants of perfect channel estimation and reception.

estimation and transmission. The average throughput figures for the 6- and 12-dB symbol power limits are 3.54 and 3.92 bits per data symbol, respectively. It can be noted that the BER floor is lower for $l=6 \mathrm{~dB}$ than for $l=12 \mathrm{~dB}$. This is because the effects of the channel variation due to the delay between the instants of channel estimation and reception in the faded subcarriers on the equalization function are much more dramatic for low-quality subbands requiring more "power boosting" than in the higher quality subcarriers. The lower the total symbol power limit $l$, the fewer the number of low-quality subcarriers used for transmission. Although not explicitly shown in the figure, we note that for both $l=6$ and $l=12 \mathrm{~dB}$, the BER performance of the blocking modem is better than that of a modem employing full channel inversion, provided that one timeslot delay is assumed. Again, the reason for this is the exclusion of the deeply faded corrupted subcarriers. If the OFDM symbol power is limited to $0 \mathrm{~dB}$, then the BER floor drops to $1.5 \cdot 10^{-6}$ at the expense of the throughput, which attains 2.5 BPS.

Fig. 26 depicts the mean OFDM symbol power histogram for this scenario. It can be seen that-as expected-the 6-dBlimited scheme has a more compact symbol power pdf, which is associated with less dramatic power fluctuation.

\section{Adaptive Modulation with Spectral Preequalization}

The preequalization algorithm discussed above inverts the channel's anticipated transfer function in order to transform the resulting channel into a Gaussian-like nonfading channel, whose SNR is dependent only on the path loss. Subband blocking has been introduced above, in order to limit the transmitter's output power, while maintaining the near-constant SNR across the used subcarriers. The preequalization algorithms discussed above do not cancel out the channel's path loss but rely on the receiver's gain control algorithm to automatically account for the channel's average path loss.

We have seen in Section VI on adaptive modulation algorithms that maintaining Gaussian channel characteristics is not the most efficient way of exploiting the channel's time-variant capacity. If maintaining a constant data throughput is not required by the rest of the communications system, then a fixed BER scheme in conjunction with error correction coding can assist in maximizing the system's throughput. The results presented for the target-BER adaptive modulation scheme in Fig. 25(b) showed that for the particular turbo-coding scheme used an uncoded BER of $1 \%$ resulted in error-free channel coded data transmission, and that for an uncoded target BER of $10 \%$, the turbo-decoded data BER was below $10^{-5}$. We have seen that it was impossible to exactly reach the anticipated uncoded target BER with the adaptive modulation algorithm, since the adaptation algorithm operated in discrete steps between modulation modes. 
Table 4

Required Target SNR Levels for $1 \%$ and $10 \%$ Target BER for the Different Modulation Schemes over an AWGN Channel

\begin{tabular}{l|c|c|c}
\hline target BER & $10^{-4}$ & $10^{-2}$ & $10^{-1}$ \\
\hline SNR(BPSK)[dB] & 8.4 & 4.33 & -0.85 \\
SNR(QPSK)[dB] & 11.42 & 7.34 & 2.16 \\
SNR(16QAM)[dB] & 18.23 & 13.91 & 7.91 \\
\hline
\end{tabular}

Combining the target-BER adaptive modulation scheme and spectral preequalization allows the transmitter to react to the channel's time- and frequency-variant nature, in order to fine-tune the behavior of the adaptive modem in fading channels. It also allows the transmitter to invest the energy that is not used in "no transmission" subbands into the other subbands without affecting the equalization at the receiver.

The combined algorithm for adaptive modulation with spectral preequalization described here does not intend to invert the channel's transfer function across the OFDM symbol's range of subcarriers; it is therefore not a pure preequalization algorithm. Instead, the aim is to transmit a subband's data symbols at a power level that ensures a given target SNR at the receiver, that is constant for all subcarriers in the subband, which in turn results in the required BER. Clearly, the receiver has to anticipate the different relative power levels for the different modulation modes, so that error-free demodulation of the multilevel modulation modes employed can be ensured.

The joint adaptation algorithm requires the estimates of the noise floor level at the receiver as well as the channel transfer function, which includes the path loss. On the basis of these values, the necessary amplitude of $E_{n}$ required to transmit a data symbol over the subcarrier $n$ for a given subcarrier SNR of $\gamma_{n}$ can be calculated as follows:

$$
\left|E_{n}\right|=\frac{\sqrt{N_{0} \cdot \gamma_{n}}}{\left|\hat{H}_{n}\right|}
$$

where $N_{0}$ is the noise floor at the receiver. The phase of $E_{n}$ is used for the preequalization, and hence

$$
\angle E_{n}=-\angle \hat{H}_{n} .
$$

The target SNR of subcarrier $n, \gamma_{n}$, is dependent on the modulation mode that is signaled over the subcarrier and determines the system's target BER. We have identified three sets of target SNR values for the modulation modes, with uncoded target BER values of $1 \%$ and $10 \%$ for use in conjunction with channel coders, as well as $10^{-4}$ for transmission without channel coding. Table 4 gives an overview of these levels, which have been read from the BER performance curves of the different modulation modes in a Gaussian channel.

Fig. 27 shows the performance of the joint preequalization and adaptive modulation algorithm over the fading time-dispersive WATM channel of Fig. 3 for the set of different target BER values of Table 4, as well as the comparison curves

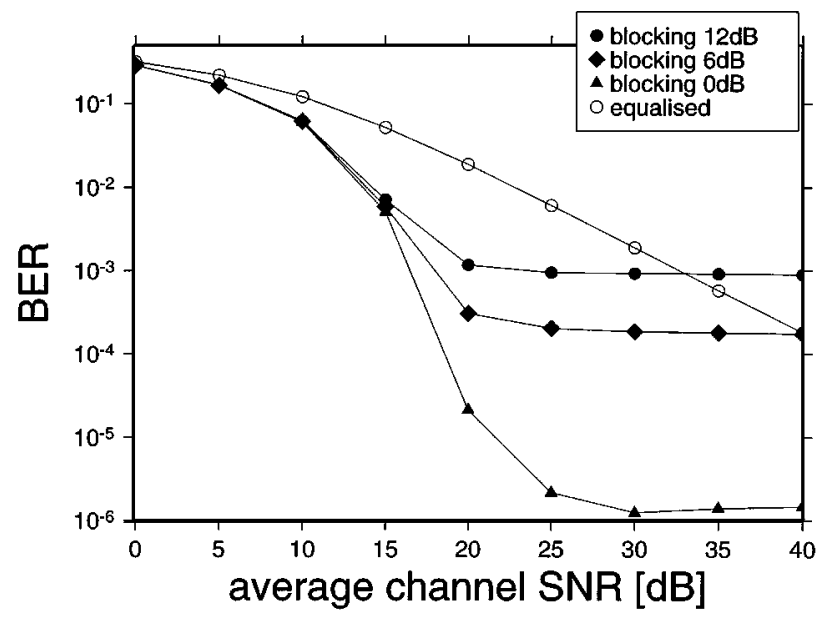

Fig. 27. OFDM symbol power histogram for 512-subcarrier 16-subband preequalization with blocking over the WATM channel of Fig. 3 using 16-QAM. The corresponding BER curves are given in Fig. 26.

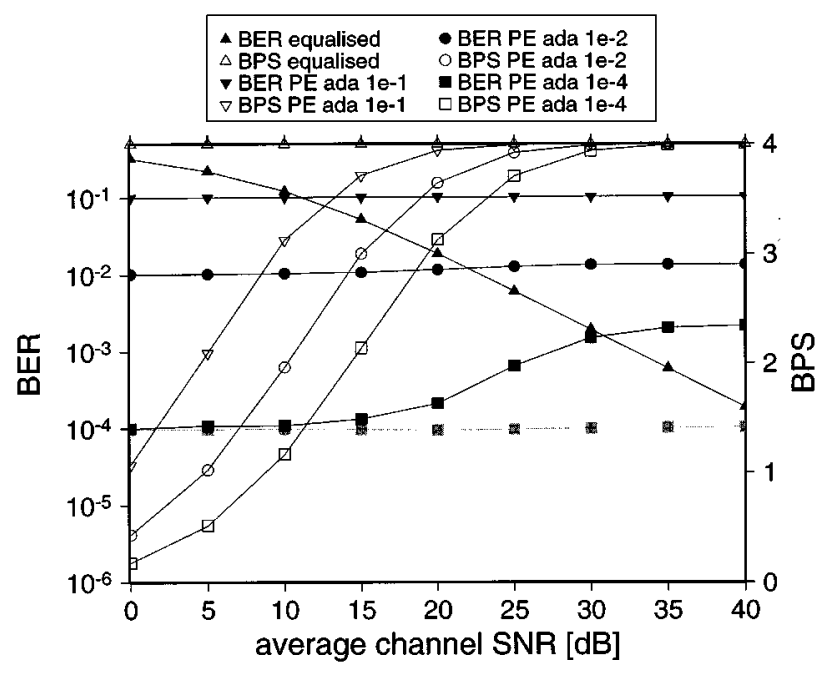

Fig. 28. BER performance and BPS throughput of the 512-subcarrier 16-subband adaptive OFDM modem with spectral preequalization over the Rayleigh-fading time-dispersive WATM channel of Fig. 3, and that of the perfectly equalized 16-QAM modem. The half-tone BER curve gives the performance of the adaptive modem for a target BER of $10^{-4}$ with no delay between channel estimation and transmission, while the other results assume one timeslot delay between up-link and down-link.

of the perfectly equalized 16-QAM modem under the same channel conditions. It can be seen that the BER achieved by the system is close to the BER targets. Specifically, for a target BER of $10 \%$, no perceptible deviation from the target has been recorded, while for the lower BER targets the deviations increase for higher channel SNR's. For a target BER of $1 \%$, the highest measured deviation is at the SNR of 40 $\mathrm{dB}$, where the recorded BER is $1.36 \%$. For the target BER of $10^{-4}$, the BER deviation is small at $0 \mathrm{~dB}$ SNR, but at an SNR of $40 \mathrm{~dB}$, the experimental BER is $2.2 \cdot 10^{-3}$. This increase of the BER with increasing SNR is due to the rapid channel variations in the deeply faded subcarriers, which are increasingly used at higher SNR values. The half-tone curve in the figure denotes the system's performance, if no delay is present between the channel estimation and the transmission. 


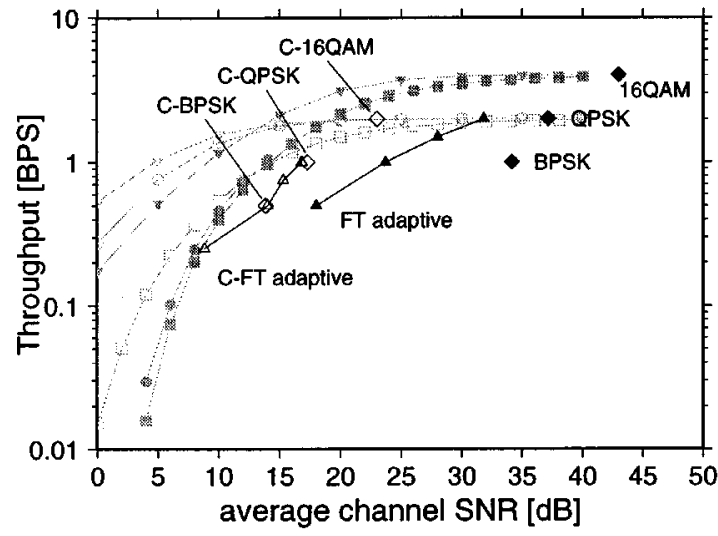

(a)

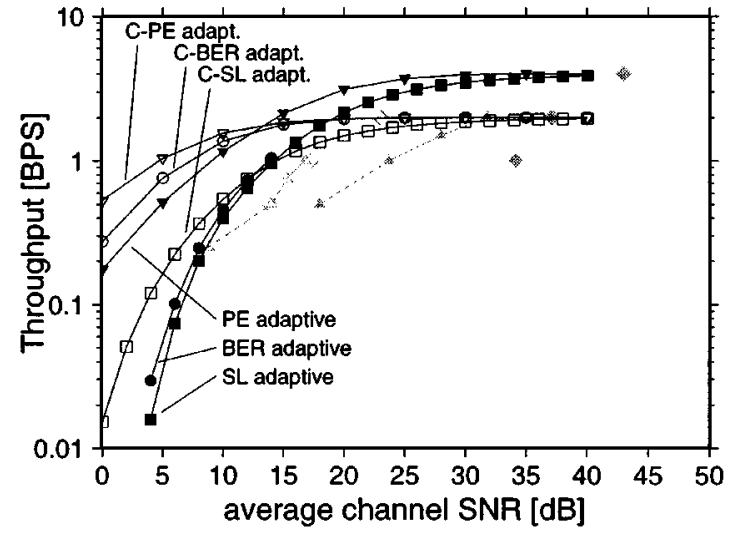

(b)

Fig. 29. BPS throughput versus average channel SNR for nonadaptive and adaptive modulation as well as for preequalized adaptive techniques, for a data BER of $10^{-4}$. Note that for the coded schemes the achieved BER values are lower than $10^{-4}$. (a) fixed-throughput systems: coded (C-) and uncoded BPSK, QPSK, 16-QAM, and fixed-throughput (FT) adaptive modulation. (b) Variable-throughput systems: coded (C-) and uncoded switching level adaptive (SL), target-BER adaptive (BER) and preequalized adaptive (PE) systems. Note that the separately plotted variable-throughput graph also shows the lightly shaded benchmark curves of the complementary fixed-rate schemes and vice versa.

In this case, the simulated BER shows only very little deviation from the target BER value. This is consistent with the behavior of the full channel inversion preequalizing modem.

\section{SUMMARY AND DISCUSSIONS}

We commenced with a historical perspective on OFDM transmissions with reference to the literature of the past 30 years. The advantages and disadvantages of various OFDM techniques were considered briefly, and the expected performance was characterized for the sake of illustration in the context of WATM systems. Our discussions deepened as we approached the subject of adaptive subcarrier modem mode allocation and channel coding. Here we would like close with a brief discussion comparing the various coded and uncoded, fixed, and adaptive OFDM modems and identify future research issues.

Specifically, Fig. 29 compares the different adaptive modulation schemes discussed. The comparison graph is split into two sets of curves, depicting the achievable data throughput for a data BER of $10^{-4}$ highlighted for the fixed-throughput systems in Fig. 29(a), and for the time-variant-throughput systems in Fig. 29(b).

The fixed-throughput systems-highlighted in black in Fig. 29(a) - comprise the nonadaptive BPSK, QPSK, and 16-QAM modems, as well as the fixed-throughput adaptive scheme, for both coded and uncoded applications. The nonadaptive modems' performance is marked on the graph as diamonds, and it can be seen that the uncoded fixed schemes require the highest channel SNR of all reviewed transmission methods to achieve a data BER of $10^{-4}$. Channel coding employing the turbo-coding schemes considered dramatically improved the SNR requirements, at the expense of half the data throughput. The uncoded FT adaptive scheme, marked by the filled triangles, yielded consistently worse data throughput than the coded (C-) fixed modulation schemes C-BPSK, C-QPSK, and C-16-QAM, with its throughput being about half the coded fixed scheme's at the same SNR values. The coded FT-adaptive (C-FT) system, however, delivered a fairly similar throughput to the C-BPSK and C-QPSK transmissions, and were capable of delivering a BER of $10^{-4}$ for SNR values down to about $9 \mathrm{~dB}$.

The variable-throughput schemes, highlighted in Fig. 29(b), outperformed the comparable fixed-throughput algorithms [110]. For high SNR values, all uncoded schemes' performance curves converged to a throughput of 4 bits/symbol, which was equivalent to 16-QAM transmission. The coded schemes reached a maximal throughput of 2 BPS. Of the uncoded schemes, the "data" switching-level (SL) and target-BER adaptive modems delivered a similar BPS performance, with the target-BER scheme exhibiting slightly better throughput than the SL adaptive modem. The adaptive modem employing preequalization (PE) significantly outperformed the other uncoded adaptive schemes and offered a throughput of 0.18 BPS at an SNR of $0 \mathrm{~dB}$, although its crest-factor PDF is slightly less attractive.

The coded transmission schemes suffered from limited throughput at high SNR values, since the half-rate channel coding limited the data throughput to 2 BPS. For low SNR values, however, the coded schemes offered better performance than the uncoded schemes, with the exception of the "speech" SL-adaptive coded scheme, which was outperformed by the uncoded PE-adaptive modem. The poor performance of the coded SL-scheme can be explained by the lower uncoded target BER of the "speech" scenario, which was $1 \%$, in contrast to the $10 \%$ uncoded target BER for the coded BER- and PE-adaptive schemes. The coded PE-adaptive modem outperformed the target-BER adaptive scheme, thanks to its more accurate control of the uncoded BER, leading to a higher throughput for low SNR values.

It is interesting to observe that for the given set of four modulation modes, the uncoded PE-adaptive scheme was close in performance to the coded adaptive schemes; and that for SNR values of more than $14 \mathrm{~dB}$, it outperformed all 
other studied schemes. It is clear, however, that the coded schemes would benefit from higher order modulation modes or higher rate channel codecs at high SNR, which would allow these modems to increase the data throughput further when the channel conditions allow.

Based on these findings, adaptive-rate channel coding is an interesting area for future research in conjunction with adaptive OFDM, adaptive beam-stearing, and interference cancellation. Provided that these OFDM enhancements reach a similar state of maturity to the OFDM components used in the current standard digital audio and video broadcasting, WATM, ADSL, and power-line communications proposals, OFDM is likely to find a range of further attractive applications in wireless and wireline communications both for businesses and in the home.

\section{ACKNOWLEDGMENT}

The constructive critique of the anonymous reviewers is gratefully acknowledged.

\section{REFERENCES}

[1] L. Hanzo, W. Webb, and T. Keller, Single- and Multi-Carrier Quadrature Amplitude Modulation. Chichester: Wiley/IEEE Press, 1999. Sample chapters: [Online]. Available: HTTP: http://www-mobile.ecs.soton.ac.uk).

[2] R. Steele and L. Hanzo, Eds., Mobile Radio Communications, 2nd ed. New York: IEEE Press/Wiley, 1999. Sample chapters: [Online]. Available: HTTP: http://www-mobile.ecs.soton.ac.uk)..

[3] P. A. Bello, "Selective fading limitations of the KATHRYN modem and some system design considerations," IEEE Trans. Commun. Technol., vol. COM-13, pp. 320-333, Sept. 1965.

[4] M. S. Zimmermann and A. L. Kirsch, "The AN/GSC-10/KATHRYN variable rate data modem for HF radio," IEEE Trans. Commun. Technol., vol. COM-15, pp. 197-205, Apr. 1967.

[5] E. N. Powers and M. S. Zimmermann, "A digital implementation of a multichannel data modem," in Proc. IEEE Int. Conf. Commun. . Philadelphia, PA, 1968.

[6] R. W. Chang, "Synthesis of band-limited orthogonal signals for multichannel data transmission," Bell Syst. Tech. J., vol. 45, pp. 1775-1796, Dec. 1966.

[7] R. W. Chang and R. A. Gibby, "A theoretical study of performance of an orthogonal multiplexing data transmission scheme," IEEE Trans. Communication Technology, vol. COM-16, pp. 529-540, Aug. 1968.

[8] B. R. Saltzberg, "Performance of an efficient parallel data transmission system," IEEE Trans. Commun. Technol., vol. COM-15, pp. 805-813, Dec. 1967.

[9] S. B. Weinstein and P. M. Ebert, "Data transmission by frquencydivision multiplexing using the discrete fourier transform," IEEE Trans. Commun. Technol., vol. COM-19, pp. 628-634, Oct. 1971.

[10] B. Hirosaki, "An analysis of automatic equalizers for orthogonally multiplexed QAM systems," IEEE Trans. Commun., vol. COM-28, pp. 73-83, Jan. 1980.

[11] A. Peled and A. Ruiz, "Frequency domain data transmission using reduced computational complexity modems," in Proc. ICASSP, 1980, pp. 964-967.

[12] B. Hirosaki, "An orthogonally multiplexed QAM system using the discrete fourier transform," IEEE Trans. Commun., vol. COM-29, pp. 982-989, July 1981.

[13] H. J. Kolb, "Untrsuchungen über ein digitales Mehrfrequensverfahren zur Datenübertragung," Auspewählte Arbeiten über Nachrichtensysteme, no. 50, 1982.

[14] H. W. Schüßler, "Ein digitales Mehrfrequenzverfahren zur Datenübertragung," in Stand und Entwicklungsaussichten der Datenund Teielcommunikation, Professoren Konferenz, Darmstadt, 1983, pp. 179-196.

[15] K. Preuss, "Ein Parallelverfahren zur schellen Datenübertragung im Ortsnetz," Auspewählte Arbeiten über Nachrichtensystemc, no. 56, 1984.
[16] R. Rückriem, "Realisierung und meßtechnische Untersuchung an einem digitalen Parellelverfahren zur Datenübertragung im Fernsprechkanal," Ausgewähite Arbeiten über Nachrichtensysteme, no. $59,1985$.

[17] I. Kalet, "The multitone channel," IEEE Trans. Commun., vol. 37, pp. 119-124, Feb. 1989.

[18] L. J. Cimini, "Analysis and simulation of a digital mobile channel using orthogonal frequency division multiplexing," IEEE Trans. Commun., vol. COM-33, pp. 665-675, July 1985.

[19] K. Fazel and G. Fettweis, Eds., Multi-Carrier Spread-Spectrum. Norwell, MA: Kluwer, 1997.

[20] F. Claßen and H. Meyr, "Synchronization algorithms for an OFDM system for mobile communications," in Codierung für Quelle, Kanal und Übertragung. Berlin: VDE-Verlag, 1994, pp. 105-113.

[21] — - "Frequency synchronization algorithms for OFDM systems suitable for communication over frequency selective fading channels," in Proc. IEEE Vehicular Technology Conf., 1994, pp. $1655-1659$.

[22] S. J. Shepherd, P. W. J. van Eetvelt, C. W. Wyatt-Millington, and S. K. Barton, "Simple coding scheme to reduce peak factor in QPSK multicarrier modulation," Electron. Lett., vol. 31, pp. 1131-1132, July 1995.

[23] A. E. Jones, T. A. Wilkinson, and S. K. Barton, "Block coding scheme for reductioon of peak to mean envelope power ratio of multicarrier transmission schemes," Electron. Lett., vol. 30, pp. 2098-2099, 1994.

[24] D. Wulich, "Reduction of peak to mean ratio of multicarrier modulation by cyclic coding," Electron. Lett., vol. 32, pp. 432-433, 1996.

[25] S. H. Muller and J. B. Huber, "Vergleich von OFDM-Verfahren mit reduzierter Spitzenleistung," in 2. OFDM-Fachpespräch in Braunschweig, 1997.

[26] M. Pauli and H-P. Kuchenbecker, "Neue Aspekte zur Reduzierung der durch Nichtlinearitäten hervorgerufenen Außerbandstrahlung eines OFDM-Signals," in 8. OFDM-Fachpesprach in Braunschweig, 1997.

[27] T. May and H. Rohling, "Reduktion von Nachbarkanalstörungen in OFDM-Funkübertragungssystemen," in 2. OFDM-Fachpespräch in Braunschweig, 1997.

[28] D. Wulich, "Peak factor in orthogonal multicarrier modulation with variable levels," Electron. Lett., vol. 32, no. 20, pp. 1859-1861, 1996.

[29] H. Schmidt and K.-D. Kammeyer, "Adaptive Subträgerselektion zur Reduktion des Crestfaktors bei OFDM," in 3. OFDM Fachpespräch in Braunschweig, 1998.

[30] R. Dinis and A. Gusmão, "Performance evaluation of OFDM transmission with conventional and 2-branch combining power amplification schemes," in Proc. IEEE Globecom, 1996, pp. 734-739.

[31] R. Dinis, P. Montezuma, and A. Gusmão, "Performance trade-offs with quasilinearly amplified OFDM through a 2-branch combining technique," in Proc. VTC, 1996, pp. 899-903.

[32] R. Dinis, A. Gusmão, and J. Fernandes, "Adaptive transmission techniques for the mobile broadband system," in Proc. ACTS Summit, Aalborg, Denmark, Oct. 1997, pp. 757-762.

[33] B. Daneshrad, J. L. J. Cimini, and M. Carloni, "Clustered-ofdm transmitter implementation," in Proc. 7th IEEE Conf. Personal Indoor and Mobile Radio Communications, Taipei, R.O.C., Oct. 1996, pp. 1064-1068.

[34] M. Okada, H. Nishijima, and S. Komaki, "A maximum likelihood decision based nonlinear distortion compensator for multi-carrier modulated signals," IEICE Trans. Commun., vol. E81B, no. 4, pp. 737-744, 1998.

[35] R. Dinis and A. Gusmão, "Performance evaluation of a multicarrier modulation technique allowing strongly nonlinear amplification," in Proc. ICC, 1998, pp. 791-796.

[36] M. V. B. T. Pollet and M. Moeneclaey, "BER sensitivity of PFDM systems to carrier frequency offset and wiener phase noise," IEEE Trans. Commun., vol. 43, pp. 191-193, Feb./Mar./Apr. 1995.

[37] H. Nikookar and R. Prasad, "On the sensitivity of multicarrier transmission over multipath channels to phase noise and frequency offset," in Proc. 7th IEEE Conf. Personal Indoor and Mobile Radio Communications, Taipei, R.O.C., Oct. 1996, pp. 68-72.

[38] J. Kuronen, V.-P. Kassila, and A. Mammela, "An all-digital symbol tracking algorithm in an OFDM system by using the cyclic prefix," in Proc. ACTS Mobile Telecommunications Summit, Granada, Spain, Nov. 1996, pp. 340-345. 
[39] M. Kiviranta and A. Mammela, "Coarse frame.synchronization structures in OFDM," in Proc. ACTS Mobile Telecommunications Summit, Granada, Spain, Nov. 1996, pp. 464-470.

[40] Z. Li and A. Mammela, "An all digital frequency synchronization scheme for OFDM systems," in Proc. IEEE Int. Symp. PIMRC'97, Helsinki, Finland, Sept. 1997, pp. 327-331.

[41] W. D. Warner and C. Leung, "Ofdm/fm frame synchronization for mobile radio data communication," IEEE Trans. Veh. Technol., vol. 42, pp. 302-313, Aug. 1993.

[42] H. Sari, G. Karam, and I. Jeanclaude, "Transmission techniques for digital terrestrial TV broadcasting," IEEE Commun. Mag., pp. 100-109, Feb. 1995.

[43] P. H. Moose, "UA technique for orthogonal frequency division multiplexing frequency offset correction," IEEE Trans. Commun., vol. 42, pp. 2908-2914, Oct. 1994.

[44] K. Bruninghaus and H. Rohling, "Verfahren zur Rahmensynchronization in einem OFDM-System," in 3. OFDM Fachgespräch in Braunschweig, 1998.

[45] F. Daffara and O. Adami, "A new frequency detector for orthogonal multicarrier transmission techniques," in Proc. IEEE 15th Vehicular Technology Conf., Chicago, IL, July 15-28, 1995, pp. 804-809.

[46] M. Sandell, J.-J. van de Beek, and P. Borjesson, "Timing and frequency synchronization in OFDM systems using the cyclic prefix," in Proc. Int. Symp. Synchronization, Essen, Germany, Dec. 14-15, 1995, pp. 16-19.

[47] N. Yee, J.-P. Linnartz, and G. Fettweis, "Multicarrier CDMA in indoor wireless radio networks," in Proc. PIMRC'9S, 1993, pp. 109-113.

[48] A. Chouly, A. Brajel, and S. Jourdan, "Orthogonal multicarrier techniques applied to direct sequence spread spectrum CDMA systems," in Proc. IEEE GLOBECOM '93, Houston, TX, Nov. 1993, pp. 1723-1728.

[49] G. Fettweis, A. Bahai, and K. Anvari, "On multi-carrier code division multiple access (mc-cdma) modem design," in Proc. IEEE VTC, 1994, pp. 1670-1674.

[50] K. Fazel and L. Papke, "On the performance of convolutionallycoded CDMA/OFDM for mobile communication system," in Proc. PIMRC'93, 1993, pp. 468-472.

[51] R. Prasad and S. Hara, "Overview of multicarrier CDMA," IEEE Commun. Mag., pp. 126-133, Dec. 1997.

[52] B.-J. Choi, E.-L. Kuan, and L. Hanzo, "Crest-factor study of MC-CDMA and OFDM," in Proc. VTC, Amsterdam, the Netherlands, Sept. 1999.

[53] Y. Li and N. R. Sollenberger, "Interference suppression in OFDM systems using adaptive antenna arrays," in Proc. IEEE Globecom 98, vol. 1, Sydney, Australia, Nov. 8-12, 1998, pp. 213-218.

[54] - "Adaptive antenna arrays for OFDM systems with cochannel interference," IEEE Trans. Commun., vol. 47, pp. 217-229, Feb. 1999.

[55] Y. Li, L. J. Cimini, and N. R. Sollenberger, "Robust channel estimation for OFDM systems with rapid dispersive fading channels," IEEE Trans. Commun., vol. 46, pp. 902-915, Apr. 1998.

[56] L. Lin, J. L. J. Cimini, and J. C.-I. Chuang, "Ibrbo codes for OFDM with antenna diversity," in Proc. IEEE Vehicular Technology Conf., Houston, TX, May 16-19, 1999.

[57] C. K. Kim, S. Choi, and Y. S. Cho, "Adaptive beamforming for an OFDM system," in Proc. IEEE Vehicular Technology Conf., Houston, TX, May 16-19, 1999.

[58] M. Munster, T. Keller, and L. Hanzo, "Co-channel interference suppression assisted adaptive OFDM in interference limited environments," in Proc. IEEE Vehicular Technology Conf., Amsterdam, the Netherlands, Sept. 19-22, 1999.

[59] F. Muller-Römer, "Directions in audio broadcasting," J. Audio Eng. Soc., vol. 41, pp. 158-173, Mar. 1993.

[60] G. Plenge, "DAB - A new radio broadcbsting system-State of development and ways for its introduction," Rundfunitechnische Mitteilungen, vol. 35, no. 2, p. 45, 1991.

[61] M. Alard and R. Lassalle, "Principles of modulation and channel coding for digital broadcasting for mobile receivers," BBU Review, pp. 47-69, Aug. 1987.

[62] Proc. 1st Int. Symp. DAB, Montreux, June 1992.

[63] ETSI, "Radio broadcasting systems: Digital Audio Broadcasting (DAB) to mobile, portable and fied receduers,", ETS 300 401, Aug. 1997.

[64] ETSI, "Digital Video Broadcasting (DVB): Framing structure, channel coding and modulation for digital terrestrial television,", EN 300 744, Aug. 1997.
[65] S. O'Leary and D. Priestly, "Mobile broadcasting of DVB-T signals," IEEE Trans. Broadcasting, vol. 44, pp. 346-352, Sept. 1998.

[66] W.-C. Lee, H.-M. Park, K.-J. Kang, and K.-B. Kim, "Performance analysis of Viterbi decoder using channel state information in COFDM system," IEEE Trans. Broadcasting, vol. 44, pp. 488-496, Dec. 1998.

[67] S. O'Leary, "Hierarchical transmission and COFDM systems," IEEE Trans. Broadcasting, vol. 43, pp. 166-174, June 1997.

[68] L. Thibault and M. T. Le, "Performance evaluation of COFDM for digital audio broadcasting_-Part I: Parametric study," IEEE Trans. Broadcasting, vol. 43, pp. 64-75, Mar. 1997.

[69] P. S. Chow, J. C. Tu, and J. M. Cioffi, "A discrete multitone transceiver system for HDSL applications," IEEE J. Select. Areas Commun., vol. 9, pp. 895-908, Aug. 1991.

[70] - "Performance evaluation of a multichannel transceiver system for ADSL and VHDSL services," IEEE J. Select. Areas Commun., vol. 9, pp. 909-919, Aug. 1991.

[71] K. Sistanizadeh, P. S. Chow, and J. M. Cioffi, "Multi-tone transmission for asymmetric digital subscriber lines (ADSL)," in Proc. IEEE ICC98, 1993, pp. 756-760.

[72] ANSI, "Asymmetric digital subscriber line (ADSL) metallic interface,", ANSI/TIEI./9J-007, Aug. 1997.

[73] A. G. Burr and P. A. Brown, "Application of OFDM to powerline telecommunications," in Proc. 3rd Int. Symp. Power-Line Communications, Lancaster, Mar. 30-Apr. 11999.

[74] M. Deinzer and M. Stoger, "Integrated PLC-modem based on OFDM," in Proc. 3rd Int. Symp. Power-Line Communications, Lancaster, Mar. 30-Apr. 11999.

[75] R. Prasad and H. Harada, "A novel OFDM based wireless ATM system for future broadband multimedia communications," in Proc. ACTS Summit, Aalborg, Denmark, Oct. 1997, pp. 757-762.

[76] C. Ciotti and J. Borowski, "The AC006 MEDIAN project-Overview and state-of-the-art," in Proc. ACTS Summit, Granada, Spain, Nov. 1996, pp. 362-367.

[77] J. Borowski, S. Zeisberg, J. Hübner, K. Koora, E. Bogenfeld, and B. Kull, "Performance of OFDM and comparable single carrier system in MEDIAN demonstrator 60GHz channel," in Proc. ACTS Summit, Aalborg, Denmark, Oct. 1997, pp. 653-658.

[78] M. G. D. Benedetto, P. Mandarini, and L. Piazzo, "Effects of a mismatch in the in-phase and in-quadrature paths, and of phase noise, in QDCPSK-OFDM modems," in Proc. ACTS Summit, Aalborg, Denmark, Oct. 1997, pp. 769-774.

[79] T. Rautio, M. Pietikainen, J. Niemi, J. Rautio, K. Rautiola, and A. Mammela, "Architecture and implementation of the $150 \mathrm{Mbit} / \mathrm{s}$ OFDM modem (invited paper)," in Proc. IEEE Benelux Joint Chapter on Communications and Vehicular Technology 6th Symp. Vehicular Technology and Communications, Helsinki, Finland, Oct. 1998, p. 11.

[80] J. Ala-Laurila and G. Awater, "The MAGIC WAND-wireless ATM network demonstrator system," in Proc. ACTS Summit, Aalborg, Denmark, Oct. 1997, pp. 356-362.

[81] J. Aldis, E. Busking, T. Kleijne, R. Kopmeiners, R. van Nee, R. Mann-Pelz, and T. Mark, "Magic into reality, building the WAND modem," in Proc. ACTS Summit, Aalborg, Denmark, Oct. 1997, pp. 775-780.

[82] E. Hallmann and H. Rohling, "OFDM-vorschläge fur UMTS," in OFDM Fachgespräch in Braunschweig, 1998.

[83] "Universal mobile telecommunications system (UMTS); UMTS terrestrial radio access (UTRA); concept evaluation,” ETSI, Tech. Rep. TR 101 146, 1997.

[84] CEPT Rec. TIR 22-06, Harmonized Radio Frequency Bands for HIPERLAN Systems, ETSI, Jan. 1998.

[85] Broadband Radio Access Networks (BRAN); High Performance Radio Local Area Networks (HIPERLAN) Type 2, System Overview, ETSI TR 101 683, v0.1.2, 1999.

[86] N. R. Prasad and H. Teunissen, "A state-of-the-art of Hiperlan/2," in Proc. VTC. Amsterdam, the Netherlands, Sept. 19-22, 1999, pp. 2661-2665.

[87] J. A. C. Bingham, "Multicarrier modulation for data transmission: An idea whose time has come," IEEE Commun. Magazine, vol. 28 , pp. 5-14, May 1990.

[88] R. van Nee and R. Prasad, OFDM for Wireless Multimedia Communications. Norwood, MA: Artech House, 2000.

[89] J. K. Cavers, "An analysis of pilot symbol assisted modulation for Rayleigh fading channels," IEEE Trans. Veh. Technol., vol. 40, no. 4, pp. 686-693, 1991.

[90] J. G. Proakis, Digital Communications, 2nd ed. London, U.K.: McGraw-Hill, 1989. 
[91] K. Fazel, S. Kaiser, P. Robertson, and M. J. Ruf, "A concept of digital terrestrial television broadcasting," in Wireless Personal Commun., 1995, vol. 2, pp. 9-27.

[92] J. A. C. Bingham, "Method and apparatus for correcting for clock and carrier frequency offset, and phase jitter in multicarrier modems," U.S. Patent 5206 886, Apr. 27, 1993.

[93] T. de Couasnon, R. Monnier, and J. B. Rault, "Ofdm for digital TV broadcasting," Signal Process., vol. 39, pp. 1-32, 1994.

[94] A. García and M. Calvo, "Phase noise and sub-carrier spacing effects on the performance of an OFDM communications system," IEEE Commun. Lett., vol. 2, pp. 11-13, Jan. 1998.

[95] W. P. Robins, Phase Noise in Signal Sources, ser. IKE Telecommunication Series: Peregrinus, 1982, vol. 9.

[96] T. Keller and L. Hanzo, "Orthogonal frequency division multiplex synchronization techniques for wireless local area networks," in Proc. 7th IEEE Conf. Personal Indoor and Mobile Radio Communications, Taipei, R.O.C., Oct. 1996, pp. 963-967.

[97] R. Steele and W. Webb, "Variable rate QAM for data transmission over Rayleigh fading channels," in Wireless. Calgary, Alta., Canada, 1991, pp. 1-14.

[98] H. Matsuoka, S. Sampei, N. Morinaga, and Y. Kamio, "Adaptive modulation system with variable coding rate concatenated code for high quality multi-media communications systems," in Proc. 46th IEEE Vehicular Technology Conf., 1996, pp. 478-491.

[99] S.-G. Chua and A. Goldsmith, "Variable-rate variable-power MQAM for fading channels," in Proc. 46th IEEE Vehicular Technology Conf., 1996, pp. 815-819.

[100] D. A. J. Pearce, A. G. Burr, and T. C. Tozer, "Comparison of countermeasures against slow rayleigh fading for TDMA systems," in Inst. Elect. Eng. Colloquium Advanced TDMA Techniques and Applications, London, Oct. 28, 1996, Colloq. Digest 1996/234.

[101] V. K. N. Lau and M. D. Macleod, "Variable rate adaptive trellis coded QAM for high bandwidth effciency applications in Rayleigh fading channels," in Proc. IEEE Vehicular Technology Conf., 1998, pp. 348-352.

[102] J. M. Torrance and L. Hanzo, "Latency and networking aspects of adaptive modems over slow indoors rayleigh fading channels," IEEE Trans. Veh. Technol., vol. 48, no. 4, pp. 1237-1251.

[103] J. M. Torrance, L. Hanzo, and T. Keller, "Interference aspects of adaptive modems over slow rayleigh fading channels," IEEE Trans. Veh. Technol., vol. 48, no. 5, Sept. 1999.

[104] A. Czylwik, "Adaptive OFDM for wideband radio channels," in Proc. IEEE Globecom, London, U.K., 1996, pp. 713-718.

[105] P. S. Chow, J. M. Cioffi, and J. A. C. Bingham, "A practical discrete multitone transceiver loading algorithm for data transmission over spectrally shaped channels," IEEE Trans. Commun., vol. 48, pp. 772-775, 1995.

[106] J. M. Torrance, "Ataptive full response digital modulation for wireless communication environments," Ph.D. dissertation, University of Southampton, Southampton, U.K., Mar. 1997.

[107] K. Miya, O. Kato, K. Homma, T. Kitade, M. Hayashi, and T. Ue, "Wideband CDMA systems in TDD-mode operation for IMT-2000," IEICE Trans. Commun., vol. E81-B, pp. 1317-1326, July 1998.

[108] O. Kato, K. Miya, K. Homma, T. Kitade, M. Hayashi, and M. Watanabe, "Experimental performance results of coherent wideband DS-CDMA with TDD scheme," IEICE Trans. Commun., vol. E81-B, pp. 1337-1344, July 1998.
[109] T. Keller and L. Hanzo, "Adaptive orthogonal frequency division multiplexing schemes," in Proc. ACST Summit, Rhodos, Greece, June 1998, pp. 794-799.

[110] T. Keller and L. Hanzo, "Blind-detection assisted sub-band adaptive turbo-coded OFDM schemes," in Proc. VTC, Houston, TX, June 1999, pp. 489-493.

[111] J. M. Torrance and L. Hanzo, "Optimization of switching levels for adaptive modulation in slow Rayleigh fading," Electron Lett., vol. 32, pp. 1167-1169, June 20, 1996.

[112] C. Berrou, A. Glavieux, and P. Thitimajshima, "Near Shannon limit error-correcting coding and decoding: Turbo-codes," in Proc. ICC, 1993, pp. 1064-1070.

[113] L. R. Bahl, J. Cocke, F. Jelinek, and J. Raviv, "Optimal decoding of linear codes for minimizing symbol error rate," IEEE Trans. Inform. Theory, pp. 284-287, Mar. 1974.

[114] T. Keller and L. Hanzo, "Sub-band adaptive pre-equalised OFDM transmission," in Proc. VTC. Amsterdam, the Netherlands, Sept. 19-22, 1999, pp. 334-338.

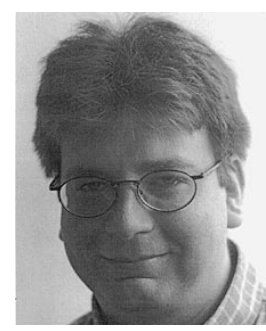

Thomas Keller studied electrical engineering at the University of Karlsruhe, Ecole Superieure d'Ingenieurs en Electronique et Electrotechnique, Paris, and the University of Southampton, U.K.. He received the Dipl.-Ing. degree in 1995 and the Ph.D. degree in mobile communications in 1999.

Between 1995 and 1999, he was with the Wireless Multimedia Communications Group, University of Southampton. His current areas of interest include adaptive OFDM transmission, wide-band channel estimation, and error correction coding. He recently joined Ubinetics, Cambridge, U.K.

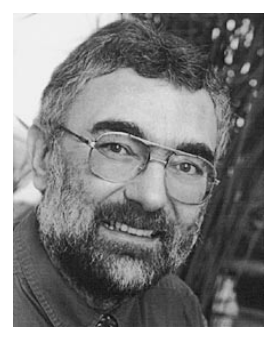

Lajos Hanzo graduated in electronics in 1976 and received the Ph.D. degree in 1983.

During his 24-year career in telecommunications, he has held various research and academic posts in Hungary, Germany, and the United Kingdom. Since 1986, he has been with the Department of Electronics and Computer Science, University of Southampton, U.K., and has been a Consultant to Multiple Access Communications Ltd., U,K. Currently, he holds the Chair in Telecommunications. He is a coauthor of five books on mobile radio communications. He has published more than 200 research papers, organized and chaired conference sessions, presented overview lectures, and been awarded a number of distinctions. Currently, he is managing a research team, working on a range of research projects in the field of wireless multimedia communications under the auspices of the Engineering and Physical Sciences Research Council (EPSRC) U.K., the European IST Programme and the Mobile Virtual Center of Excellence (VCE). He also provides a range of industrial training courses. 